\title{
Analysis of a Class of Fractional Nonlinear Multidelay Differential Systems
}

\author{
Zhuoyan Gao, ${ }^{1}$ JinRong Wang, ${ }^{2}$ and Yong Zhou ${ }^{3,4}$ \\ ${ }^{1}$ College of Applied Mathematics, Shanxi University of Finance and Economics, Taiyuan, Shanxi 030031, China \\ ${ }^{2}$ Department of Mathematics, Guizhou University, Guiyang, Guizhou 550025, China \\ ${ }^{3}$ Department of Mathematics, Xiangtan University, Xiangtan, Hunan 411105, China \\ ${ }^{4}$ Nonlinear Analysis and Applied Mathematics (NAAM) Research Group, Faculty of Science, King Abdulaziz University, \\ Jeddah 21589, Saudi Arabia
}

Correspondence should be addressed to Yong Zhou; yzhou@xtu.edu.cn

Received 31 July 2017; Accepted 30 August 2017; Published 15 October 2017

Academic Editor: Antonio Iannizzotto

Copyright (c) 2017 Zhuoyan Gao et al. This is an open access article distributed under the Creative Commons Attribution License, which permits unrestricted use, distribution, and reproduction in any medium, provided the original work is properly cited.

We address existence and Ulam-Hyers and Ulam-Hyers-Mittag-Leffler stability of fractional nonlinear multiple time-delays systems with respect to two parameters' weighted norm, which provides a foundation to study iterative learning control problem for this system. Secondly, we design PID-type learning laws to generate sequences of output trajectories to tracking the desired trajectory. Two numerical examples are used to illustrate the theoretical results.

\section{Introduction}

Fractional differential equations have been used to deal with many problems from physics, engineering, and other fields. For some basic results in the theory of fractional differential equations, one can read the monographs [1-3] or the survey [4] and reference therein. Recently, considerable attention has been given to the control and stability of fractional differential equations; one can refer to [5-25] via Ulam's type stability concepts and the references therein. We also note that there are some contributions on Mittag-Leffler stability of fractional order systems and stabilization [26-29]. We remark that there are some difference between the concept of MittagLeffler stability and Ulam-Hyers-Mittag-Leffler stability. The concept of Mittag-Leffler stability of solution follows the idea of stability of zero solution for the classical ODEs and gives an estimate inequality for the norm of solution via MittagLeffler function. The concept of Ulam-Hyers-Mittag-Leffler stability follows the idea of Ulam-Hyers stability of functional equations and gives an approximate relation via small parameter and Mittag-Leffler function between the solution of equations and the solution of inequalities, which is a special case of Ulam-Hyers-Rassias stability. That is, we try to find a solution of approximate inequalities close to the solution of the original equations in the sense of Ulam-Hyers-MittagLeffler stability. The main idea for this concept will provide an approach to find the explicit solution. However, there are only few works on existence and Ulam's type stability for the nonlinear fractional time-delays differential equations.

Iterative learning control has become a popular strategy in the intelligent control community since it was proposed by Uchiyama [30] and developed by Arimoto et al. [31]. Recently, iterative learning control problems of P-type, D-type, I-type, or their combination schemes have been widely applied to various types of repetitive or batch dynamical systems (see, e.g., [32-38]). The problem on designing an ILC for uncertain plants with time-delays has not been fully investigated, and only a limited number of the results are available so far (see, e.g., [39-41]). However, most of the existing literatures focus on iterative learning control of the nonlinear fractional differential system without time-delays, especially multiple time-delays. Note that PID-type ILC learning algorithm is one of the popular updating laws. The advantage of PID-type ILC learning algorithm is simple and very easy to be realized in tracking problem. The disadvantage of PID-type ILC is that the error characterization for the signal is not the best 
and there is not a uniform method to design the weighting coefficients.

Delay systems are widely used to model dynamical systems in many scientific and engineering areas, for example, biology, climatology, and economy. Comparing with systems with single delay, systems with multidelay are more realistic models in the interacting complex systems. In fact, dynamics of multifeedback systems are representative examples of the multidelay systems.

Motivated by $[15,42]$, we firstly discuss existence, UlamHyers stability, and Ulam-Hyers-Mittag-Leffler stability of solutions to fractional order nonlinear Cauchy problems with multiple time-delays of the form:

$$
\begin{aligned}
& \left({ }^{c} D_{0+}^{\alpha} x\right)(t) \\
& =f\left(t, x(t), x\left(t-\tau_{1}\right), \ldots, x\left(t-\tau_{m}\right)\right) \\
& +I\left(t, x(t), x\left(t-\xi_{1}\right), \ldots, x\left(t-\xi_{n}\right)\right) u(t), \\
& \quad t \in[0, T], 0<\alpha<1, \\
& x(t)=\psi(t), \\
& \quad t \in[-a, 0], a=\max \left\{\tau_{1}, \ldots, \tau_{m}, \xi_{1}, \ldots, \xi_{n}\right\},
\end{aligned}
$$

where $T$ is a positive constant; ${ }^{c} D_{0+}^{\alpha}$ is the Caputo fractional derivative of order $\alpha$ with the lower limit zero; $u \in$ $C([0, T], \mathbb{R}) ; \tau_{1}, \ldots, \tau_{m}, \xi_{1}, \ldots, \xi_{n}$ are positive constant timedelays; $x(t) \in \mathbb{R} ; \psi$ is the initial continuous function of the system in $t \in[-a, 0] ; f \in C([0, T] \times \underbrace{\mathbb{R} \times \cdots \mathbb{R}}_{n}, \mathbb{R})$; and $I \in C([0, T] \times \underbrace{\mathbb{R} \times \cdots \mathbb{R}}_{n}, \mathbb{R})$.

Secondly, we turn to study PID-type ILC learning algorithm of the following fractional order nonlinear system with output equation:

$$
\begin{aligned}
& \left({ }^{c} D_{0+}^{\alpha} x_{k}\right)(t) \\
& =f\left(t, x_{k}(t), x_{k}\left(t-\tau_{1}\right), \ldots, x_{k}\left(t-\tau_{m}\right)\right) \\
& +I\left(t, x_{k}(t), x_{k}\left(t-\xi_{1}\right), \ldots, x_{k}\left(t-\xi_{n}\right)\right) u_{k}(t), \\
& t \in[0, T], 0<\alpha<1, \\
& x_{k}(t)=\psi_{k}(t) \text {, } \\
& t \in[-a, 0], a=\max \left\{\tau_{1}, \ldots, \tau_{m}, \xi_{1}, \ldots, \xi_{n}\right\}, \\
& y_{k}(t)=g\left(t, x_{k}(t)\right)+d \int_{0}^{t} u_{k}(s) d s, \quad t \in[0, T],
\end{aligned}
$$

where $k$ denotes the $k$ th learning iteration; $u_{k}(t) \in \mathbb{R}$ and $y_{k}(t) \in \mathbb{R}$ are the states and control input and output of the system, respectively; $\psi_{k}$ is the initial continuous function of the system in $t \in[-a, 0] ; f, I$, and $u_{k}$ are given continuous functions in $[0, T]$; and $g:[0, T] \times \mathbb{R} \rightarrow \mathbb{R}$ is a continuous function.

The rest of the paper is organized as follows. Section 2 collects some notations and preparation results. Section 3 presents existence and uniqueness of solutions and shows Ulam-Hyers stability and Ulam-Hyers-Mittag-Leffler stability of solutions by using Picard operator method. Section 4 presents convergence result for PID-type ILC updating law. Section 5 gives two illustrative examples.

\section{Preliminaries}

Denote $X:=C([-a, T], \mathbb{R})$ as the Banach space of continuous functions from $[-a, T] \rightarrow \mathbb{R}$ endowed with the $(\lambda, \alpha)$-norm $\|x\|_{\lambda, \alpha}=\max _{t \in[-a, T]} e^{-\lambda t^{\alpha}}|x(t)|(x \in C([-a, T], \mathbb{R}), \lambda>$ $0,0<\alpha<1)$.

Definition 1 (see [2]). The Riemann-Liouville fractional integrals $I_{a+}^{\alpha} f$ are defined by

$$
\left(I_{a+}^{\alpha} f\right)(x):=\frac{1}{\Gamma(\alpha)} \int_{a}^{x} \frac{f(t)}{(x-t)^{1-\alpha}} d t, \quad(x>a ; \alpha>0),
$$

and the Riemann-Liouville fractional derivatives $D_{a+}^{\alpha} f$ are defined by

$$
\begin{aligned}
{ }^{L}\left(D_{a+}^{\alpha} f\right)(x): & =\left(\frac{d}{d x}\right)^{n}\left(I_{a+}^{n-\alpha} f\right)(x) \\
= & \frac{1}{\Gamma(n-\alpha)}\left(\frac{d}{d x}\right)^{n} \int_{a}^{x} \frac{f(t)}{(x-t)^{\alpha-n+1}} d t, \\
& (x>a ; \alpha>0 ; n=[\alpha]+1),
\end{aligned}
$$

where $\Gamma(\cdot)$ is Gamma function.

Definition 2 (see [2]). The Caputo derivative of order $\gamma$ for a function $f:[a, \infty) \rightarrow R$ can be written as

$$
\begin{aligned}
{ }^{c} D_{a+}^{\gamma} f(t)={ }^{L} D_{a+}^{\gamma}\left(f(t)-\sum_{k=a}^{n-1} \frac{t^{k}}{k !} f^{(k)}(a)\right) & \\
& t>0, n-1<\gamma<n .
\end{aligned}
$$

Definition 3 (see [43]). Let $(X, d)$ be a metric space. An $A$ : $X \rightarrow X$ is a Picard operator if there exists $x^{*} \in X$ such that (i) $F_{A}=x^{*}$, where $F_{A}=\{x \in X: A(x)=x\}$ is the fixed point set of $A$; (ii) the sequence $\left(A^{n}\left(x_{0}\right)\right)_{n \in N}$ converges to $x^{*}$ for all $x_{0} \in X$.

Lemma 4 (see [43]). Let $(X, d, \leq)$ be an ordered metric space and $A: X \rightarrow X$ be an increasing Picard operator $\left(F_{A}=x^{*}\right)$. Then, for $x \in X, x \leq A(x)$ implies $x \leq x_{A}^{*}$.

The following Gronwall inequalities will be used in the sequel.

Lemma 5 (see [44, Lemma 3.1]). Let $u(t)$ be a continuous function on $t \in[0, T]$ and let $v(t-\tau)$ be continuous and nonnegative on the triangle $0 \leq \tau \leq t$. Moreover, let $w(t)$ be a positive continuous and nondecreasing function on $t \in[0, T]$. If

$$
u(t) \leq w(t)+\int_{0}^{t} v(t-\tau) u(\tau) d \tau, \quad t \in[0, T]
$$

then

$$
u(t) \leq w(t) e^{\int_{0}^{t} v(t-\tau) d \tau}, \quad t \in[0, T]
$$


Lemma 6 (see [44, Lemma 7.1.1]). Let $z, \omega:[0, T) \rightarrow[0, \infty)$ be continuous functions where $T \leq \infty$. If $\omega$ is nondecreasing and there are constants $\kappa \geq 0$ and $0<\alpha<1$ such that

$$
z(t) \leq \omega(t)+\kappa \int_{0}^{t}(t-s)^{\alpha-1} z(s) d s, \quad t \in[0, T),
$$

then

$$
\begin{array}{r}
z(t) \leq \omega(t)+\int_{0}^{t}\left(\sum_{n=1}^{\infty} \frac{(\kappa \Gamma(\alpha))^{n}}{\Gamma(n \alpha)}(t-s)^{n \alpha-1} \omega(s)\right) d s, \\
t \in[0, T) .
\end{array}
$$

Remark 7 (see [44]). Under the hypothesis of Lemma 6, let $\omega(t)$ be a nondecreasing function on $[0, T)$. Then we have $z(t) \leq \omega(t) E_{\alpha}\left(\kappa \Gamma(\alpha) t^{\alpha}\right)$.

By [45, Lemma 2.12], one can adopt the similar idea to prove the following result.

Lemma 8. Let $0<\alpha<1$ and $\lambda>0$. Set

$$
Z:=\int_{0}^{t}(t-s)^{\alpha-1} e^{\lambda s^{\alpha}} d s, \quad t \in[0, T], T>0 .
$$

Then

$$
\begin{aligned}
& Z \leq \frac{t^{\alpha} e^{\lambda t^{\alpha}}}{\alpha([\lambda]+1)^{\alpha}}, \quad \text { if }[\lambda]=0, \\
& Z \leq \frac{e^{\lambda t^{\alpha}}\left(1+t^{\alpha}\right)}{\alpha[\lambda]^{\alpha}}, \quad \text { if }[\lambda] \geq 1,
\end{aligned}
$$

where $T$ is a positive number and $[\lambda]$ denotes the integer part of $\lambda$.

Proof. For completeness we supply the proofs. Denote

$$
\begin{aligned}
& Z_{1}:=\sum_{k=1}^{[\lambda]} \int_{(k-1) t /([\lambda]+1)}^{k t /([\lambda]+1)}(t-s)^{\alpha-1} e^{\lambda s^{\alpha}} d s, \\
& Z_{2}:=\int_{[\lambda] t /([\lambda]+1)}^{t}(t-s)^{\alpha-1} e^{\lambda s^{\alpha}} d s .
\end{aligned}
$$

Case 1. If $[\lambda]=0$ then $Z=Z_{2}$. Obviously,

$$
\begin{aligned}
Z_{2} & =\int_{[\lambda] t /([\lambda]+1)}^{t}(t-s)^{\alpha-1} e^{\lambda s^{\alpha}} d s \\
& \leq e^{\lambda t^{\alpha}} \int_{[\lambda] t /([\lambda]+1)}^{t}(t-s)^{\alpha-1} d s=\frac{t^{\alpha} e^{\lambda t^{\alpha}}}{\alpha([\lambda]+1)^{\alpha}} .
\end{aligned}
$$

Case 2. If $[\lambda] \geq 1$ then $Z=Z_{1}+Z_{2}$. Obviously,

$$
\begin{aligned}
& \int_{(k-1) t /([\lambda]+1)}^{k t /([\lambda]+1)}(t-s)^{\alpha-1} e^{\lambda s^{\alpha}} d s \\
& \quad \leq \int_{(k-1) t /([\lambda]+1)}^{k t /[\lambda]+1)}\left(\frac{s([\lambda]+1)}{k}-s\right)^{\alpha-1}
\end{aligned}
$$

$$
\begin{aligned}
& \cdot e^{\lambda s^{\alpha}} d s\left(s \leq \frac{k t}{[\lambda]+1} \Longrightarrow t \geq \frac{s([\lambda]+1)}{k}\right) \\
& \leq\left(\frac{([\lambda]+1)}{k}-1\right)^{\alpha-1} \int_{(k-1) t /([\lambda]+1)}^{k t /([\lambda]+1)} s^{\alpha-1} e^{\lambda s^{\alpha}} d s \\
& \leq \frac{1}{\lambda \alpha}\left(\frac{([\lambda]+1)}{k}-1\right)^{\alpha-1}\left(e^{\lambda(k t /([\lambda]+1))^{\alpha}}\right. \\
& \left.-e^{\lambda((k-1) t /([\lambda]+1))^{\alpha}}\right) .
\end{aligned}
$$

Hence, we get

$$
\begin{aligned}
Z_{1} & :=\sum_{k=1}^{[\lambda]} \int_{(k-1) t /([\lambda]+1)}^{k t /(\lambda]+1)}(t-s)^{\alpha-1} e^{\lambda s^{\alpha}} d s \\
& \leq \sum_{k=1}^{[\lambda]} \frac{1}{\lambda \alpha}\left(\frac{([\lambda]+1)}{k}-1\right)^{\alpha-1}\left(e^{\lambda(k t /([\lambda]+1))^{\alpha}}\right. \\
& \left.-e^{\lambda\left(((k-1) t /([\lambda]+1))^{\alpha}\right.}\right) \leq \frac{1}{\lambda \alpha}\left[[\lambda]^{\alpha-1}\left(e^{\lambda(t /([\lambda]+1))^{\alpha}}-1\right)\right. \\
& +\left(\frac{([\lambda]+1)}{2}-1\right)^{\alpha-1}\left(e^{\lambda(2 t /([\lambda]+1))^{\alpha}}-e^{\lambda(t /([\lambda]+1))^{\alpha}}\right) \\
& +\cdots+\left(\frac{([\lambda]+1)}{[\lambda]}-1\right)^{\alpha-1} \\
& \left.\cdot\left(e^{\lambda([\lambda] t /([\lambda]+1))^{\alpha}}-e^{\lambda(([\lambda]-1) t /([\lambda]+1))^{\alpha}}\right)\right] \\
& \leq \frac{1}{\lambda \alpha}\left[\left(\frac{([\lambda]+1)}{1}-1\right)^{\alpha-1}\left(e^{\lambda(t /([\lambda]+1))^{\alpha}}-1\right)\right. \\
& +\left(\frac{([\lambda]+1)}{2}-1\right)^{\alpha-1}\left(e^{\lambda(2 t /([\lambda]+1))^{\alpha}}-1\right)+\cdots \\
& +e^{\lambda([\lambda] t /([\lambda]+1))^{\alpha}} \leq \frac{e^{\lambda t^{\alpha}}}{\alpha[\lambda]^{\alpha}} . \\
& \left.+\left(\frac{([\lambda]+1)}{[\lambda]}-1\right)^{\alpha-1}\left(e^{\lambda([\lambda] t /([\lambda]+1))^{\alpha}}-1\right)\right] \leq \frac{1}{\lambda \alpha} \\
& +[\lambda][\lambda]^{1-\alpha}\left(e^{\lambda([\lambda] t /([\lambda]+1))^{\alpha}}-1\right) \\
& \frac{1}{\alpha[\lambda]^{\alpha-1}}\left(e^{\lambda([\lambda] t /([\lambda]+1))^{\alpha}}-1\right) \leq \frac{1}{\alpha[\lambda]^{\alpha-1}} \\
& \\
&
\end{aligned}
$$

Furthermore, we can get

$$
Z=Z_{1}+Z_{2} \leq \frac{e^{\lambda t^{\alpha}}}{\alpha[\lambda]^{\alpha}}+\frac{t^{\alpha} e^{\lambda t^{\alpha}}}{\alpha([\lambda]+1)^{\alpha}} \leq \frac{e^{\lambda t^{\alpha}}\left(1+t^{\alpha}\right)}{\alpha[\lambda]^{\alpha}} .
$$

The proof is finished. 


\section{Existence and Stability Results}

We introduce the following assumptions:

$\left(H_{1}\right)$ Assume that $f \in C([0, T] \times \underbrace{\mathbb{R} \times \cdots \mathbb{R}}_{n}, \mathbb{R}), I \in$ $C([0, T] \times \underbrace{\mathbb{R} \times \cdots \mathbb{R}}_{n}, \mathbb{R})$, and $u \in C([0, T], \mathbb{R})$. In addition, set $u_{b}=\max _{t \in[0, T]}|u(t)|$.

$\left(H_{2}\right)$ For arbitrary $x_{1}, x_{2} \in C([-a, T], \mathbb{R})$, there exist positive constants $L_{f}, L_{I}$ such that

$$
\begin{aligned}
& \mid f\left(t, x_{2}(t), x_{2}\left(t-\tau_{1}\right), \ldots, x_{2}\left(t-\tau_{m}\right)\right) \\
& \quad-f\left(t, x_{1}(t), x_{1}\left(t-\tau_{1}\right), \ldots, x_{1}\left(t-\tau_{m}\right)\right) \mid \\
& \quad \leq L_{f}\left(\left|x_{2}(t)-x_{1}(t)\right|\right. \\
& \left.\quad+\left|\sum_{l=1}^{m}\left(x_{2}\left(t-\tau_{l}\right)-x_{1}\left(t-\tau_{l}\right)\right)\right|\right) \\
& \mid I\left(t, x_{2}(t), x_{2}\left(t-\xi_{1}\right), \ldots, x_{2}\left(t-\xi_{m}\right)\right) \\
& \quad-I\left(t, x_{1}(t), x_{1}\left(t-\xi_{1}\right), \ldots, x_{1}\left(t-\xi_{m}\right)\right) \mid
\end{aligned}
$$

$$
\begin{aligned}
& \leq L_{I}\left(\left|x_{2}(t)-x_{1}(t)\right|\right. \\
& \left.+\left|\sum_{l=1}^{n}\left(x_{2}\left(t-\xi_{l}\right)-x_{1}\left(t-\xi_{l}\right)\right)\right|\right) .
\end{aligned}
$$

$\left(H_{3}\right)$ Suppose the following inequalities hold:

$$
\begin{gathered}
\frac{c T^{\alpha}}{\Gamma(\alpha+1)}<1, \quad[\lambda]=0, \\
\frac{c\left(1+T^{\alpha}\right)}{\Gamma(\alpha+1)[\lambda]^{\alpha}}<1, \quad[\lambda] \geq 1,
\end{gathered}
$$

where $c=L_{f}(m+1)+L_{I} b_{u}(n+1)$.

Theorem 9. Assume that $\left(H_{1}\right),\left(H_{2}\right)$, and $\left(H_{3}\right)$ are satisfied. Then problem (1) has a unique solution in $C([-a, T], \mathbb{R})$.

Proof. Define an operator $A: X \rightarrow X(X:=C([-a, T], \mathbb{R}))$ as follows:

$$
\begin{aligned}
& A(x)(t) \\
& =\left\{\begin{array}{lr}
\psi(t), & t \in[-a, 0], \\
\psi(0)+\frac{1}{\Gamma(\alpha)} \int_{0}^{t}(t-s)^{\alpha-1}\left(f\left(s, x(s), x\left(s-\tau_{1}\right), \ldots, x\left(s-\tau_{m}\right)\right)+I\left(s, x(s), x\left(s-\xi_{1}\right), \ldots, x\left(s-\xi_{n}\right)\right) u(s)\right) d s, & t \in[0, T] .
\end{array}\right.
\end{aligned}
$$

Next, we show that $A$ defined in (19) is a contraction mapping on $X$ with respect to the previous $(\lambda, \alpha)$-norm $\|\cdot\|_{\lambda, \alpha}$. For all $t \in[-a, 0]$ and $x(t), z(t) \in X$, we have $\| A(x)(t)-$ $A(z)(t) \|=0$. This yields that $\|A(x)-A(z)\|_{\lambda, \alpha}=0$.

For any $t \in[0, T]$ and $x, z \in X$, according to $\left(H_{2}\right)$, we have

$$
\begin{aligned}
& |A(x)(t)-A(z)(t)| \leq \frac{1}{\Gamma(\alpha)} \int_{0}^{t}(t-s)^{\alpha-1} \\
& \quad \cdot \mid f\left(s, x(s), x\left(s-\tau_{1}\right), \ldots, x\left(s-\tau_{m}\right)\right) \\
& \quad-f\left(s, z(s), z\left(s-\tau_{1}\right), \ldots, z\left(s-\tau_{m}\right)\right) \mid \\
& \quad+\mid\left(I\left(s, x(s), x\left(s-\xi_{1}\right), \ldots, x\left(s-\xi_{n}\right)\right)\right. \\
& \left.\quad-I\left(s, z(s), z\left(s-\xi_{1}\right), \ldots, z\left(s-\xi_{n}\right)\right)\right)|| u(s) \mid d s \\
& \quad+\frac{L_{f}}{\Gamma(\alpha)} \int_{0}^{t}(t-s)^{\alpha-1}(|x(s)-z(s)| \\
& \left.\quad+\left|\sum_{l=1}^{m}\left(x\left(s-\tau_{l}\right)-z\left(s-\tau_{l}\right)\right)\right|\right) d s+\frac{L_{I} b_{u}}{\Gamma(\alpha)} \\
& \quad+\mid \sum_{0}^{t}(t-s)^{\alpha-1}(|x(s)-z(s)| \\
& \left.\left.\left.\quad \mid s-\xi_{l}\right)-z\left(s-\xi_{l}\right)\right) \mid\right) d s .
\end{aligned}
$$

Let

$$
\begin{aligned}
G(\theta)=|x(t-\theta)-z(t-\theta)| & \\
\theta & \in\left\{\tau_{1}, \ldots, \tau_{m}, \xi_{1}, \ldots, \xi_{n}\right\} .
\end{aligned}
$$

$$
\begin{gathered}
|A(x)(t)-A(z)(t)| \leq \frac{L_{f}}{\Gamma(\alpha)} \int_{0}^{t}(t-s)^{\alpha-1} \\
\cdot\left(|x(s)-z(s)|+\left|\sum_{l=1}^{m} G\left(\tau_{l}\right)\right|\right) d s+\frac{L_{I} b_{u}}{\Gamma(\alpha)} \\
\cdot \int_{0}^{t}(t-s)^{\alpha-1}\left(|x(s)-z(s)|+\left|\sum_{l=1}^{n} G\left(\xi_{l}\right)\right|\right) d s \\
\leq \frac{L_{f}}{\Gamma(\alpha)} \int_{0}^{t}(t-s)^{\alpha-1} \\
\cdot e^{\lambda s^{\alpha}} e^{-\lambda s^{\alpha}}\left(|x(s)-z(s)|+\sum_{l=1}^{m} G\left(\tau_{l}\right)\right) d s
\end{gathered}
$$




$$
\begin{aligned}
& +\frac{L_{I} b_{u}}{\Gamma(\alpha)} \int_{0}^{t}(t-s)^{\alpha-1} \\
& \cdot e^{\lambda s^{\alpha}} e^{-\lambda s^{\alpha}}\left(|x(s)-z(s)|+\sum_{l=1}^{n} G\left(\xi_{l}\right)\right) d s .
\end{aligned}
$$

Consider $G(\cdot)$ defined in (21); we have

$$
\begin{aligned}
& \int_{0}^{t}(t-s)^{\alpha} e^{\lambda s^{\alpha}} e^{-\lambda s^{\alpha}} G(\theta) d s \\
& =\int_{0}^{t}(t-s)^{\alpha} e^{\lambda s^{\alpha}} e^{-\lambda s^{\alpha}}|x(s-\theta)-z(s-\theta)| d s \\
& \leq \begin{cases}\int_{0}^{t}(t-s)^{\alpha} e^{\lambda s^{\alpha}} e^{-\lambda s^{\alpha}}|\psi(s)-\psi(s)| d s=0, & t \in[0, \theta], \\
\int_{0}^{t}(t-s)^{\alpha} e^{\lambda s^{\alpha}}\|x-z\|_{\lambda, \alpha} d s, & t \in[\theta, T],\end{cases} \\
& \leq \int_{0}^{t}(t-s)^{\alpha} e^{\lambda s^{\alpha}} d s\|x-z\|_{\lambda, \alpha} .
\end{aligned}
$$

Substituting (23) into (22), using Lemma 8, we can obtain

$$
\begin{aligned}
& |A(x)(t)-A(z)(t)| \leq \frac{L_{f}}{\Gamma(\alpha)} \int_{0}^{t}(t-s)^{\alpha-1} \\
& \cdot e^{\lambda s^{\alpha}} e^{-\lambda s^{\alpha}}\left(|x(s)-z(s)|+\sum_{l=1}^{m} G\left(\tau_{l}\right)\right) d s \\
& +\frac{L_{I} b_{u}}{\Gamma(\alpha)} \int_{0}^{t}(t-s)^{\alpha-1} \\
& \cdot e^{\lambda s^{\alpha}} e^{-\lambda s^{\alpha}}\left(|x(s)-z(s)|+\sum_{l=1}^{n} G\left(\xi_{l}\right)\right) d s \\
& \quad \leq \frac{L_{f}(m+1)+L_{I} b_{u}(n+1)}{\Gamma(\alpha)} \int_{0}^{t}(t-s)^{\alpha-1} e^{\lambda s^{\alpha}} d s \| x \\
& -z \|_{\lambda, \alpha} \quad[\lambda] \geq 1 . \\
& \quad \leq\left\{\begin{array}{l}
\frac{c T^{\alpha} e^{\lambda t^{\alpha}}}{\Gamma(\alpha+1)(1+[\lambda])^{\alpha}}\|x-z\|_{\lambda, \alpha}, \quad[\lambda]=0, \\
\frac{c\left(1+T^{\alpha}\right) e^{\lambda t^{\alpha}}}{\Gamma(\alpha+1)[\lambda]^{\alpha}}\|x-z\|_{\lambda, \alpha},
\end{array}\right.
\end{aligned}
$$

$$
\begin{aligned}
& \left({ }^{c} D_{0+}^{\alpha} x\right)(t) \\
& =f\left(t, x(t), x\left(t-\tau_{1}\right), \ldots, x\left(t-\tau_{m}\right)\right) \\
& +I\left(t, x(t), x\left(t-\xi_{1}\right), \ldots, x\left(t-\xi_{n}\right)\right) u(t), \\
& \quad t \in[0, T], 0<\alpha<1, \\
& x(t)=\bar{z}(t), \\
& \quad t \in[-a, 0], a=\max \left\{\tau_{1}, \ldots, \tau_{m}, \xi_{1}, \ldots, \xi_{n}\right\} .
\end{aligned}
$$

Remark 11. A function $\bar{z} \in X$ is a solution of inequality (26) if and only if there exists a function $\bar{w} \in X$ (which depend on

(i) $|\bar{w}(t)| \leq \epsilon, t \in[-a, T]$.

(ii) ${ }^{c} D_{0, t}^{\alpha} \bar{z}(t)=f\left(t, \bar{z}(t), \bar{z}\left(t-\tau_{1}\right), \ldots, \bar{z}\left(t-\tau_{m}\right)\right)+$ $I\left(t, \bar{z}(t), \bar{z}\left(t-\xi_{1}\right), \ldots, \bar{z}\left(t-\xi_{n}\right)\right) u(t)+\bar{w}(t), t \in$ $[0, T], 0<\alpha<1$

Theorem 12. Assume that $\left(H_{1}\right),\left(H_{2}\right)$, and $\left(H_{3}\right)$ are satisfied; then (1) is Ulam-Hyers stable.

Proof. Let $\bar{z} \in C([-a, T], \mathbb{R})$ be a solution of inequality (26) and $x(t)$ be a solution of

Then

$$
\begin{aligned}
& x(t) \\
& = \begin{cases}\bar{z}(t), & t \in[-a, 0], \\
\bar{z}(0)+\frac{1}{\Gamma(\alpha)} \int_{0}^{t}(t-s)^{\alpha-1}\left[f\left(s, x(s), x\left(s-\tau_{1}\right), \ldots, x\left(s-\tau_{m}\right)\right)+I\left(s, x(s), x\left(s-\xi_{1}\right), \ldots, x\left(s-\xi_{n}\right)\right) u(s)\right] d s, & t \in[0, T] .\end{cases}
\end{aligned}
$$


Obviously,

$$
\begin{aligned}
&{ }^{c} D_{0, t}^{\alpha} \bar{z}(t) \\
&= f\left(t, \bar{z}(t), \bar{z}\left(t-\tau_{1}\right), \ldots, \bar{z}\left(t-\tau_{m}\right)\right) \\
&+I\left(t, \bar{z}(t), \bar{z}\left(t-\xi_{1}\right), \ldots, \bar{z}\left(t-\xi_{n}\right)\right) u(t) \\
&+\bar{w}(t), \quad t \in[0, T],
\end{aligned}
$$

which can be turned to

$$
\begin{aligned}
\bar{z}(t) & =\bar{z}(0)+\frac{1}{\Gamma(\alpha)} \int_{0}^{t}(t-s)^{\alpha-1} \\
\cdot & {\left[f\left(s, \bar{z}(s), \bar{z}\left(s-\tau_{1}\right), \ldots, \bar{z}\left(s-\tau_{m}\right)\right)\right.} \\
+ & I\left(s, \bar{z}(s), \bar{z}\left(s-\xi_{1}\right), \ldots, \bar{z}\left(s-\xi_{n}\right)\right) u(s) \\
+ & \bar{w}(s)] d s, \quad t \in[0, T]
\end{aligned}
$$

where $\bar{w} \in X$ (which depend on $\bar{z})$.
According to Remark 11, one has

$$
\begin{aligned}
& \mid \bar{z}(t)-\bar{z}(0)-\frac{1}{\Gamma(\alpha)} \int_{0}^{t}(t-s)^{\alpha-1} \\
& \cdot\left[f\left(s, \bar{z}(s), \bar{z}\left(s-\tau_{1}\right), \ldots, \bar{z}\left(s-\tau_{m}\right)\right)\right. \\
& \left.\quad-I\left(s, \bar{z}(s), \bar{z}\left(s-\xi_{1}\right), \ldots, \bar{z}\left(s-\xi_{n}\right)\right) u(s)\right] d s \mid \\
& \quad \leq \frac{1}{\Gamma(\alpha)} \int_{0}^{t}(t-s)^{\alpha-1}|\bar{w}(s)| d s \leq \frac{\epsilon}{\Gamma(\alpha)} \int_{0}^{t}(t \\
& \quad-s)^{\alpha-1} d s \leq c_{1} \epsilon, \quad c_{1}=\frac{T^{\alpha}}{\Gamma(\alpha+1)} .
\end{aligned}
$$

Therefore,

$$
\begin{aligned}
& |\bar{z}(t)-x(t)| \leq \mid \bar{z}(t)-\bar{z}(0)-\frac{1}{\Gamma(\alpha)} \int_{0}^{t}(t-s)^{\alpha-1}\left(f\left(s, \bar{z}(s), \bar{z}\left(s-\tau_{1}\right), \ldots, \bar{z}\left(s-\tau_{m}\right)\right)\right. \\
& \left.\quad+I\left(s, \bar{z}(s), \bar{z}\left(s-\xi_{1}\right), \ldots, \bar{z}\left(s-\xi_{n}\right)\right) u(s)+\bar{w}(s)\right) d s\left|+\frac{1}{\Gamma(\alpha)}\right| \int_{0}^{t}(t-s)^{\alpha-1} \\
& \quad \times\left[\left(f\left(s, x(s), x\left(s-\tau_{1}\right), \ldots, x\left(s-\tau_{m}\right)\right)+I\left(s, x(s), x\left(s-\xi_{1}\right), \ldots, x\left(s-\xi_{n}\right)\right) u(s)\right)\right. \\
& \left.\quad-\left(f\left(s, \bar{z}(s), \bar{z}\left(s-\tau_{1}\right), \ldots, \bar{z}\left(s-\tau_{m}\right)\right)+I\left(s, \bar{z}(s), \bar{z}\left(s-\xi_{1}\right), \ldots, \bar{z}\left(s-\xi_{n}\right)\right) u(s)\right)\right] d s \mid \leq c_{1} \epsilon+\int_{0}^{t}(t-s)^{\alpha-1} \\
& \quad+\mid \frac{L_{f}}{\Gamma(\alpha)}\left(|\bar{z}(s)-x(s)|+\left|\sum_{l=1}^{m}\left(\bar{z}\left(s-\tau_{l}\right)-x\left(s-\tau_{l}\right)\right)\right|\right)+\frac{L_{I} b_{u}}{\Gamma(\alpha)}(|\bar{z}(s)-x(s)| \\
& \left.\left.\quad+\left|\sum_{l=1}^{n}\left(\bar{z}\left(s-\xi_{l}\right)-x\left(s-\xi_{l}\right)\right)\right|\right)\right] d s \leq\left\{\begin{array}{c}
c T^{\alpha} \\
c_{1} \epsilon+\frac{c}{\Gamma(\alpha+1)(1+[\lambda])^{\alpha}}\|\bar{z}-x\|_{\lambda, \alpha}, \quad[\lambda]=0, t \in[0, T] \\
c_{1} \epsilon+\frac{c\left(1+T^{\alpha}\right)}{\Gamma(\alpha+1)[\lambda]^{\alpha}}\|\bar{z}-x\|_{\lambda, \alpha}, \quad[\lambda] \geq 1, t \in[0, T]
\end{array}\right.
\end{aligned}
$$

Now multiplying by the fact $e^{-\lambda t^{\alpha}}$ on both side of the above inequalities, one can derive that

$$
\begin{aligned}
\left(1-\frac{c T^{\alpha}}{\Gamma(\alpha+1)(1+[\lambda])^{\alpha}}\right)\|\bar{z}-x\|_{\lambda, \alpha} & \leq c_{1} \epsilon, \\
{[\lambda] } & =0, t \in[0, T], \\
\left(1-\frac{c\left(1+T^{\alpha}\right)}{\Gamma(\alpha+1)[\lambda]^{\alpha}}\right)\|\bar{z}-x\|_{\lambda, \alpha} & \leq c_{1} \epsilon, \\
{[\lambda] } & \geq 1, t \in[0, T] .
\end{aligned}
$$

$$
\begin{aligned}
&|\bar{z}(t)-x(t)| \leq \frac{c_{1} \epsilon e^{\lambda T^{\alpha}}}{1-c\left(1+T^{\alpha}\right) / \Gamma(\alpha+1)[\lambda]^{\alpha}} \\
&=\frac{c_{1} \epsilon \Gamma(\alpha+1)[\lambda]^{\alpha} e^{\lambda T^{\alpha}}}{\Gamma(\alpha+1)[\lambda]^{\alpha}-c\left(1+T^{\alpha}\right)}, \\
& {[\lambda] \geq 1, t \in[0, T] . }
\end{aligned}
$$

Furthermore, according to $\left(\mathrm{H}_{3}\right)$, combined with the fact of $|\bar{z}(t)-x(t)|=0, t \in[-a, 0]$, we can get

$$
|\bar{z}(t)-x(t)| \leq \widehat{c} \epsilon, \quad t \in[-a, T],
$$

where

$$
\begin{aligned}
|\bar{z}(t)-x(t)| & \leq \frac{c_{1} \epsilon e^{\lambda T^{\alpha}}}{1-c T^{\alpha} / \Gamma(\alpha+1)(1+[\lambda])^{\alpha}} \\
& =\frac{c_{1} \epsilon \Gamma(\alpha+1)(1+[\lambda])^{\alpha} e^{\lambda T^{\alpha}}}{\Gamma(\alpha+1)(1+[\lambda])^{\alpha}-c T^{\alpha}}, \\
& {[\lambda]=0, t \in[0, T], }
\end{aligned}
$$

$$
\begin{aligned}
\widehat{c}= & \max \left\{\frac{c_{1} \Gamma(\alpha+1)(1+[\lambda])^{\alpha} e^{\lambda T^{\alpha}}}{\Gamma(\alpha+1)(1+[\lambda])^{\alpha}-c T^{\alpha}},\right. \\
& \left.\frac{c_{1} \Gamma(\alpha+1)[\lambda]^{\alpha} e^{\lambda T^{\alpha}}}{\Gamma(\alpha+1)[\lambda]^{\alpha}-c\left(1+T^{\alpha}\right)}\right\} .
\end{aligned}
$$

Therefore, (1) is Ulam-Hyers stable. 
Different from the above stability result in the Theorem 12 , in the following part, we will discuss Ulam-HyersMittag-Leffler stability of (1) on the time interval $[-a, T]$. So we first introduce the following Ulam-Hyers-Mittag-Leffler stability definition.

Consider problem (1) with

$$
\begin{aligned}
& \mid{ }^{c} D_{0, t}^{\alpha} z(t)-f\left(t, z(t), z\left(t-\tau_{1}\right), \ldots, z\left(t-\tau_{m}\right)\right) \\
& \quad-I\left(t, z(t), z\left(t-\xi_{1}\right), \ldots, z\left(t-\xi_{n}\right)\right) u(t) \mid \\
& \quad \leq \epsilon E_{\alpha}\left(t^{\alpha}\right), \quad t \in[0, T], 0<\alpha<1,
\end{aligned}
$$

where $E_{\alpha}$ is the Mittag-Leffler function [2] defined by

$$
E_{\alpha}(z):=\sum_{k=0}^{\infty} \frac{z^{k}}{\Gamma(k \alpha+1)}, \quad z \in C, \quad \operatorname{Re}(\alpha)>0 .
$$

Definition 13 ([46]). Equation (1) is Ulam-Hyers-MittagLeffler stable with respect to $E_{\alpha}\left(t^{\alpha}\right)$ if there exists $c_{E_{\alpha}}>0$ such that for each $\epsilon>0$ and for each solution $z \in X$ to (38), there exists a solution $x \in X$ to (1) with

$$
|z(t)-x(t)| \leq c_{E_{\alpha}} \in E_{\alpha}\left(t^{\alpha}\right), \quad t \in[-a, T] .
$$

Remark 14 ([46]). A function $z \in X$ is a solution of inequality (38) if and only if there exists a function $w \in X$ (which depends on $z$ ) such that

(i) $|w(t)| \leq \epsilon E_{\alpha}\left(t^{\alpha}\right)$ for all $t \in[0, T]$;

(ii) ${ }^{c} D_{0, t}^{\alpha} z(t)=f\left(t, z(t), z\left(t-\tau_{1}\right), \ldots, z\left(t-\tau_{m}\right)\right)+$ $I\left(t, z(t), z\left(t-\xi_{1}\right), \ldots, z\left(t-\xi_{n}\right)\right) u(t)+w(t), t \in$ $[0, T], 0<\alpha<1$.

Theorem 15. Assume that $\left(H_{1}\right),\left(H_{2}\right)$, and $\left(H_{3}\right)$ are satisfied; then (1) is Ulam-Hyers-Mittag-Leffler stable.

Proof. Let $z \in X$ be a solution to (38) and $x \in X$ be the unique solution of the following problem:

$$
\begin{aligned}
{ }^{c} D_{0, t}^{\alpha} x(t) & \\
= & f\left(t, x(t), x\left(t-\tau_{1}\right), \ldots, x\left(t-\tau_{m}\right)\right) \\
& +I\left(t, x(t), x\left(t-\xi_{1}\right), \ldots, x\left(t-\xi_{n}\right)\right) u(t), \\
& \quad t \in[0, T], \\
x(t) & =z(t), \quad t \in[-a, 0] .
\end{aligned}
$$

Obviously,

$$
\begin{aligned}
& x(t) \\
& = \begin{cases}z(t), & t \in[-a, 0], \\
z(0)+\frac{1}{\Gamma(\alpha)} \int_{0}^{t}(t-s)^{\alpha-1}\left[f\left(s, x(s), x\left(s-\tau_{1}\right), \ldots, x\left(s-\tau_{m}\right)\right)+I\left(s, x(s), x\left(s-\xi_{1}\right), \ldots, x\left(s-\xi_{n}\right)\right) u(s) d s,\right. & t \in[0, T] .\end{cases}
\end{aligned}
$$

On the other hand, from [47, Remark 2] via Remark 14, we can know that $z$ satisfied the following inequality:

$$
\begin{aligned}
& \mid z(t)-z(0)-\frac{1}{\Gamma(\alpha)} \int_{0}^{t}(t-s)^{\alpha-1} \\
& \cdot\left[f\left(s, z(s), z\left(s-\tau_{1}\right), \ldots, z\left(s-\tau_{m}\right)\right)\right. \\
& \left.\quad+I\left(s, z(s), z\left(s-\xi_{1}\right), \ldots, z\left(s-\xi_{n}\right)\right) u(s)\right] d s \mid \\
& \leq \frac{1}{\Gamma(\alpha)} \int_{0}^{t}(t-s)^{\alpha-1}|w(s)| d s \leq \frac{\epsilon}{\Gamma(\alpha)} \int_{0}^{t}(t-s)^{\alpha-1} \\
& \cdot E_{\alpha}\left(s^{\alpha}\right) d s \leq \epsilon E_{\alpha}\left(t^{\alpha}\right), \quad t \in[0, T] .
\end{aligned}
$$

Then, for $t \in[0, T]$, according to $\left(H_{2}\right)$, we have

$$
\begin{aligned}
& |z(t)-x(t)| \leq \mid z(t)-z(0)-\frac{1}{\Gamma(\alpha)} \int_{0}^{t}(t-s)^{\alpha-1} \\
& \cdot\left(f\left(s, z(s), z\left(s-\tau_{1}\right), \ldots, z\left(s-\tau_{m}\right)\right)\right. \\
& \left.\quad+I\left(s, z(s), z\left(s-\xi_{1}\right), \ldots, z\left(s-\xi_{n}\right)\right) u(s)\right) d s \mid
\end{aligned}
$$

$$
\begin{aligned}
& +\frac{1}{\Gamma(\alpha)} \mid \int_{0}^{t}(t-s)^{\alpha-1} \\
& \cdot\left(f\left(s, z(s), z\left(s-\tau_{1}\right), \ldots, z\left(s-\tau_{m}\right)\right)\right. \\
& \left.+I\left(s, z(s), z\left(s-\xi_{1}\right), \ldots, z\left(s-\xi_{n}\right)\right) u(s)\right) d s \\
& -\int_{0}^{t}(t-s)^{\alpha-1} \\
& \cdot\left(f\left(s, x(s), x\left(s-\tau_{1}\right), \ldots, x\left(s-\tau_{m}\right)\right)\right. \\
& \left.+I\left(s, x(s), x\left(s-\xi_{1}\right), \ldots, x\left(s-\xi_{n}\right)\right) u(s)\right) d s \mid \\
& \leq \epsilon E_{\alpha}\left(t^{\alpha}\right)+\frac{1}{\Gamma(\alpha)} \int_{0}^{t}(t-s)^{\alpha-1}(\mid(f \\
& \cdot\left(s, z(s), z\left(s-\tau_{1}\right), \ldots, z\left(s-\tau_{m}\right)\right) \\
& -f\left(s, x(s), x\left(s-\tau_{1}\right), \ldots, x\left(s-\tau_{m}\right)\right) \mid \\
& +\mid I\left(s, z(s), z\left(s-\xi_{1}\right), \ldots, z\left(s-\xi_{n}\right)\right) \\
& -I\left(s, x(s), x\left(s-\xi_{1}\right), \ldots, x\left(s-\xi_{n}\right)\right) \mid
\end{aligned}
$$




$$
\begin{aligned}
& \cdot|u(s)|) d s \leq \epsilon E_{\alpha}\left(t^{\alpha}\right)+\frac{L_{f}}{\Gamma(\alpha)} \int_{0}^{t}(t-s)^{\alpha-1} \\
& \cdot(|x(s)-z(s)| \\
& \left.+\left|\sum_{l=1}^{m}\left(x\left(s-\tau_{l}\right)-z\left(s-\tau_{l}\right)\right)\right|\right) d s+\frac{L_{I} b_{u}}{\Gamma(\alpha)}
\end{aligned}
$$

$$
\begin{aligned}
& \cdot \int_{0}^{t}(t-s)^{\alpha-1}(|x(s)-z(s)| \\
& \left.+\left|\sum_{l=1}^{n}\left(x\left(s-\xi_{l}\right)-z\left(s-\xi_{l}\right)\right)\right|\right) d s .
\end{aligned}
$$

Note the fact $|z(t)-x(t)|=0, t \in[-a, 0]$. Consider the operator $F: C\left([-a, T], \mathbb{R}_{+}\right) \rightarrow C\left([-a, T], \mathbb{R}_{+}\right)$defined by

$$
\begin{aligned}
& F(\bar{x})(t) \\
& =\left\{\begin{array}{l}
0, \\
\epsilon E_{\alpha}\left(t^{\alpha}\right)+\frac{L_{f}}{\Gamma(\alpha)}\left(\int_{0}^{t}(t-s)^{\alpha-1} \bar{x}(s) d s+\int_{0}^{t}(t-s)^{\alpha-1} \sum_{l=1}^{m}\left(\bar{x}\left(s-\tau_{l}\right)\right)\right) d s+\frac{L_{I} b_{u}}{\Gamma(\alpha)}\left(\int_{0}^{t}(t-s)^{\alpha-1} \bar{x}(s) d s+\int_{0}^{t}(t-s)^{\alpha-1} \sum_{l=1}^{n}\left(\bar{x}\left(s-\xi_{l}\right)\right)\right) d s, \quad t \in[0, T],
\end{array}\right.
\end{aligned}
$$

where $\bar{x} \in C\left([-a, T], \mathbb{R}_{+}\right)$.

Next, we verify that $F$ is a Picard operator. In fact, for all $t \in[0, T]$ and arbitrary $\bar{x}, \bar{z} \in C\left([-a, T], \mathbb{R}_{+}\right)$, it follows the proof in Theorem 9; one can show that $F$ is a contraction via the $(\lambda, \alpha)$-norm on $C\left([-a, T], \mathbb{R}_{+}\right)$due to $\left(H_{3}\right)$.

Applying the Banach contraction principle to $F$, we derive that $F$ is a Picard operator and $G_{F}=\left\{x^{*}\right\}$. Then, we have $x^{*}=0$, for $t \in[-a, 0]$ and

$$
\begin{aligned}
& x^{*}(t)=\epsilon E_{\alpha}\left(t^{\alpha}\right)+\frac{L_{f}}{\Gamma(\alpha)}\left(\int_{0}^{t}(t-s)^{\alpha-1} x^{*}(s) d s\right. \\
& \left.+\int_{0}^{t}(t-s)^{\alpha-1} \sum_{l=1}^{m}\left(x^{*}\left(s-\tau_{l}\right)\right) d s\right) \\
& +\frac{L_{I} b_{u}}{\Gamma(\alpha)}\left(\int_{0}^{t}(t-s)^{\alpha-1} x^{*}(s) d s\right. \\
& \left.+\int_{0}^{t}(t-s)^{\alpha-1} \sum_{l=1}^{n}\left(x^{*}\left(s-\xi_{l}\right)\right) d s\right), \quad t \in[0, T] .
\end{aligned}
$$

We go on to verify that the solution $x^{*}$ is increasing. Now, denote $m_{1}:=\min _{s \in[0, T]}\left[x^{*}(s)+\sum_{l=1}^{m} x^{*}\left(s-\tau_{l}\right)\right] \in \mathbb{R}_{+}$and $m_{2}:=\min _{s \in[0, T]}\left[x^{*}(s)+\sum_{l=1}^{n} x^{*}\left(s-\xi_{l}\right)\right] \in \mathbb{R}_{+}$.

Then, for $0 \leq t_{1} \leq t_{2} \leq T$, we have

$$
\begin{gathered}
x^{*}\left(t_{2}\right)-x^{*}\left(t_{1}\right)=\epsilon\left[E_{\alpha}\left(t_{2}^{\alpha}\right)-E_{\alpha}\left(t_{1}^{\alpha}\right)\right] \\
+\frac{L_{f}}{\Gamma(\alpha)}\left(\int_{0}^{t_{1}}\left[\left(t_{2}-s\right)^{\alpha-1}-\left(t_{1}-s\right)^{\alpha-1}\right]\right. \\
\left.\cdot\left(x^{*}(s)+\sum_{l=1}^{m} x^{*}\left(s-\tau_{l}\right)\right) d s\right) \\
+\frac{L_{I} b_{u}}{\Gamma(\alpha)}\left(\int_{0}^{t_{1}}\left[\left(t_{2}-s\right)^{\alpha-1}-\left(t_{1}-s\right)^{\alpha-1}\right]\right.
\end{gathered}
$$

$$
\begin{aligned}
& \left.\cdot\left(x^{*}(s)+\sum_{l=1}^{n} x^{*}\left(s-\xi_{l}\right)\right) d s\right) \\
& +\frac{L_{f}}{\Gamma(\alpha)}\left(\int_{t_{1}}^{t_{2}}\left(t_{2}-s\right)^{\alpha-1}\right. \\
& \left.\cdot\left(x^{*}(s)+\sum_{l=1}^{m} x^{*}\left(s-\tau_{l}\right)\right) d s\right) \\
& +\frac{L_{I} b_{u}}{\Gamma(\alpha)}\left(\int_{t_{1}}^{t_{2}}\left(t_{2}-s\right)^{\alpha-1}\right. \\
& \left.\cdot\left(x^{*}(s)+\sum_{l=1}^{n} x^{*}\left(s-\xi_{l}\right)\right) d s\right) \geq \epsilon\left[E_{\alpha}\left(t_{2}^{\alpha}\right)\right. \\
& \left.-E_{\alpha}\left(t_{1}^{\alpha}\right)\right]+\left(\frac{L_{f} m_{1}}{\Gamma(\alpha)}+\frac{L_{I} b_{u} m_{2}}{\Gamma(\alpha)}\right) \int_{0}^{t_{1}}\left[\left(t_{2}-s\right)^{\alpha-1}\right. \\
& \left.-\left(t_{1}-s\right)^{\alpha-1}\right] d s+\left(\frac{L_{f} m_{1}}{\Gamma(\alpha)}+\frac{L_{I} b_{u} m_{2}}{\Gamma(\alpha)}\right) \\
& +\int_{t_{1}}^{t_{2}}\left(t_{2}-s\right)^{\alpha-1} d s=\epsilon\left[E_{\alpha}\left(t_{2}^{\alpha}\right)-E_{\alpha}\left(t_{1}^{\alpha}\right)\right] \\
& +\left(\frac{L_{f} m_{1}}{\Gamma(\alpha+1)}+\frac{L_{I} b_{u} m_{2}}{\Gamma(\alpha+1)}\right)\left(t_{2}^{\alpha}-t_{1}^{\alpha}\right)>0 .
\end{aligned}
$$

So, $x^{*}$ is increasing. Thus, $x^{*}(t-\theta) \leq x^{*}(t)$ due to $t-\theta \leq$ $t\left(\theta \in\left\{\tau_{1}, \ldots, \tau_{m}, \xi_{1}, \ldots, \xi_{n}\right\}\right)$ and

$$
\begin{gathered}
x^{*}(t) \leq \epsilon E_{\alpha}\left(t^{\alpha}\right)+\frac{L_{f}(1+m)}{\Gamma(\alpha)} \int_{0}^{t}(t-s)^{\alpha-1} x^{*}(s) d s \\
+\frac{L_{I} b_{u}(n+1)}{\Gamma(\alpha)} \int_{0}^{t}(t-s)^{\alpha-1} x^{*}(s) d s \leq \epsilon E_{\alpha}\left(t^{\alpha}\right) \\
+\frac{L_{f}(m+1)+L_{I} b_{u}(n+1)}{\Gamma(\alpha)} \int_{0}^{t}(t-s)^{\alpha-1} x^{*}(s) d s .
\end{gathered}
$$


Using Lemma 6, Remark 7, and the fact of $x^{*}=0$, for $t \in[-a, 0]$, we obtain

$$
\begin{aligned}
& x^{*}(t) \\
& \quad \leq \epsilon E_{\alpha}\left(t^{\alpha}\right) E_{\alpha}\left(\left(L_{f}(m+1)+L_{I} b_{u}(n+1)\right) T^{\alpha}\right) \\
& \quad \leq c_{E_{\alpha}} \epsilon E_{\alpha}\left(t^{\alpha}\right), \quad t \in[-a, T],
\end{aligned}
$$

where $c_{E_{\alpha}}=E_{\alpha}\left(\left(L_{f}(m+1)+L_{I} b_{u}(n+1)\right) T^{\alpha}\right)$.

In particular, if $\bar{x}(t)=|z(t)-x(t)|$, from (44), $\bar{x} \leq F \bar{x}$ and applying the Lemma 4 , we obtain $\bar{x} \leq x_{F}^{*}$, where $F$ is a Picard and an increasing operator. As a result, we know

$$
|z(t)-x(t)| \leq c_{E_{\alpha}} \in E_{\alpha}\left(t^{\alpha}\right), \quad t \in[-a, T] .
$$

Thus, (2) is Ulam-Hyers-Mittag-Leffler stable.

Remark 16. One can find that we use Gronwall's inequality method to derive asymptotic stability of the corresponding systems instead of using the Lyapunov direct method in $[28,29]$. We do not need to assume that Lyapunov function satisfies some certain condition, for example, [28, Theorem 5, (12)-(13)]. Next, note that $E_{\alpha}(z) \leq 1$ if $z \leq 0$; then the definition of Mittag-Leffler stability can be turned to stability of zero solutions. However, the concept of Ulam-HyersMittag-Leffler is more general since $E_{\alpha}\left(t^{\alpha}\right)$ is not necessarily less than 1 on the whole interval $[-a, T]$.

\section{PID-Type ILC}

In this section, we consider the open-loop and close-loop PID-type ILC updating laws of fractional order nonlinear system with multiple time-delays (2) via $(\lambda, \alpha)$-weighted norm $\|\cdot\|_{\lambda, \alpha}$.

4.1. Open-Loop Case. For system (2), consider the open-loop PID-type ILC updating law with initial state learning:

$$
\begin{aligned}
& x_{k+1}(0)=x_{k}(0)+\varrho e_{k}(0) \\
& u_{k+1}(t)=u_{k}(t)+\eta_{1} e_{k}(t)+\eta_{2} \dot{e}_{k}(t)+\eta_{3} \int_{0}^{t} e_{k}(s) d s,
\end{aligned}
$$

where $\varrho$ and $\eta_{i}(i=1,2,3)$ are unknown parameters to be determined. used:

For the sake of brevity, the following notations will be

$$
\begin{aligned}
f_{k}(t) & =f\left(t, x_{k}(t), x_{k}\left(t-\tau_{1}\right), \ldots, x_{k}\left(t-\tau_{m}\right)\right), \\
I_{k}(t) & =I\left(t, x_{k}(t), x_{k}\left(t-\xi_{1}\right), \ldots, x_{k}\left(t-\xi_{n}\right)\right), \\
g_{k}(t) & =g\left(t, x_{k}(t)\right) .
\end{aligned}
$$

And we denote that $\Delta u_{k}(t)=u_{k+1}(t)-u_{k}(t), \Delta x_{k}(t)=$ $x_{k+1}(t)-x_{k}(t)$, and $e_{k}(t)=y_{d}(t)-y_{k}(t)$ which is called tracking error at $k$ th repetition, where $y_{d}(t)$ denotes desired output bounded trajectory satisfying

$$
\begin{aligned}
& \left({ }^{c} D_{0+}^{\alpha} x_{d}\right)(t) \\
& =f\left(t, x_{d}(t), x_{d}\left(t-\tau_{1}\right), \ldots, x_{d}\left(t-\tau_{m}\right)\right) \\
& +I\left(t, x_{d}(t), x_{d}\left(t-\xi_{1}\right), \ldots, x_{d}\left(t-\xi_{n}\right)\right) u_{d}(t), \\
& t \in[0, T], 0<\alpha<1, \\
& x_{d}(t)=\psi_{d}(t) \\
& t \in[-a, 0], a=\max \left\{\tau_{1}, \ldots, \tau_{m}, \xi_{1}, \ldots, \xi_{n}\right\}, \\
& y_{d}(t)=g\left(t, x_{d}(t)\right)+d \int_{0}^{t} u_{d}(s) d s, \quad t \in[0, T],
\end{aligned}
$$

and $u_{d}$ is a desired control.

In this section, we imposed the following assumptions on the class of system described by (2).

$\left(H_{4}\right)$ The function $g$ is continuous and differentiable for all $x$ and $t$ with partial derivatives $g_{x}$ and $g_{t}$. For the constant $\beta_{j}, j=1,2$, set

$$
0<\beta_{1} \leq g_{x}(\cdot, x):=\frac{\partial g(\cdot, x)}{\partial x} \leq \beta_{2}
$$

$\left(H_{5}\right)$ The function $I\left(t, x(t), x\left(t-\xi_{1}\right), \ldots, x\left(t-\xi_{m}\right)\right)$ is uniformly bounded on $[0, T]$; that is, there exists $b_{I}>0$ such that $\left|I\left(t, x(t), x\left(t-\xi_{1}\right), \ldots, x\left(t-\xi_{m}\right)\right)\right| \leq b_{I}$ for any $t \in[0, T]$.

Theorem 17. Assume that $\left(H_{1}\right)-\left(H_{5}\right)$ hold. If

$$
\begin{aligned}
& \max \left\{\left|1-\beta_{1} \varrho\right|,\left|1-\beta_{2} \varrho\right|\right\} \leq r_{0}<1, \\
& r_{1}:=\left|1-d \gamma_{2}\right|+\left|d \gamma_{1} T\right|+\frac{\left|d \gamma_{3}\right| T^{2}}{2}<1,
\end{aligned}
$$

then the nonlinear fractional multiple time-delays differential system (2) with the open-loop PID-type ILC updating law (51) guarantees that $y_{k}$ tends to $y_{d}$ as $k \rightarrow \infty$ in the sense of $(\lambda, \alpha)$ norm for all $t \in[0, T]$, where $\psi_{d}(t)$ is the desired initial state on $[-a, 0]$ and $y_{d}(t)$ is the desired output trajectory.

Proof. Note that

$$
\begin{aligned}
e_{k+1}(t)= & e_{k}(t)+g_{k}(t)-g_{k+1}(t) \\
= & e_{k}(t)-g_{x}\left(\mu_{k}(t), t\right) \Delta x_{k}(t) \\
& -d \int_{0}^{t} \Delta u_{k}(s) d s,
\end{aligned}
$$

where $\mu_{k}(t)$ lies in the segment with end point $x_{k}(t)$ and $x_{k+1}(t)$ for $t \in[0, T]$.

In what follows, we show that $\left\|e_{k}\right\|_{\lambda, \alpha} \rightarrow 0$ as $k \rightarrow \infty$ for a.e. $t \in[0, T]$. By using mean value theorem we have

$$
\begin{aligned}
e_{k+1}(0) & =e_{k}(0)+y_{k}(0)-y_{k+1}(0) \\
& =e_{k}(0)-g_{x}\left(\mu_{k}(0), 0\right) \Delta x_{k}(0) .
\end{aligned}
$$


Substituting (51) into (58) and taking the absolute value, we have

$$
\begin{aligned}
\left|e_{k+1}(0)\right| & =\left|e_{k}(0)+y_{k}(0)-y_{k+1}(0)\right| \\
& =\left|e_{k}(0)-g_{x}\left(\mu_{k}(0), 0\right) \Delta x_{k}(0)\right| \\
& =\left|e_{k}(0)-g_{x}\left(\mu_{k}(0), 0\right) \varrho e_{k}(0)\right| \\
& \leq\left|1-\varrho g_{x}\left(\mu_{k}(0), 0\right)\right|\left|e_{k}(0)\right| \leq r_{0}\left|e_{k}(0)\right| .
\end{aligned}
$$

It follows from (55) that

$$
\lim _{k \rightarrow \infty}\left\|e_{k}(0)\right\|_{\lambda, \alpha}=0
$$

Now we turn to give an estimation for the upper bound of $\Delta x_{k}$. One has

$$
\begin{aligned}
& \Delta x_{k}(t)=x_{k+1}(t)-x_{k}(t)=\Delta x_{k}(0)+\frac{1}{\Gamma(\alpha)} \int_{0}^{t}(t \\
& -s)^{\alpha-1}\left(f_{k+1}(s)+I_{k+1}(s) u_{k+1}(s)-f_{k}(s)\right. \\
& \left.+I_{k}(s) u_{k}(s)\right) d s=\Delta x_{k}(0)+\frac{1}{\Gamma(\alpha)} \int_{0}^{t}(t \\
& -s)^{\alpha-1}\left(\left(f_{k+1}(s)-f_{k}(s)\right)\right. \\
& +\left(I_{k+1}(s)-I_{k}(s)\right) u_{k+1}(s) \\
& \left.+I_{k}(s)\left(u_{k+1}(s)-u_{k}(s)\right)\right) d s .
\end{aligned}
$$

Repeating the same procedure in Theorem 9, we can get

$$
\begin{aligned}
& \left|\Delta x_{k}(t)\right|=\left|x_{k+1}(t)-x_{k}(t)\right| \leq\left|\Delta x_{k}(0)\right|+\frac{1}{\Gamma(\alpha)} \int_{0}^{t}(t \\
& -s)^{\alpha-1} \mid f_{k+1}(s)+I_{k+1}(s) u_{k+1}(s)-f_{k}(s) \\
& \quad-I_{k}(s) u_{k}(s)|d s \leq| \Delta x_{k}(0) \mid+\frac{1}{\Gamma(\alpha)} \int_{0}^{t}(t \\
& \quad-s)^{\alpha-1}\left(\left|f_{k+1}(s)-f_{k}(s)\right|\right. \\
& +\left|I_{k+1}(s)-I_{k}(s)\right|\left|u_{k+1}(s)\right| \\
& \left.+\left|I_{k}(s)\right|\left|u_{k+1}(s)-u_{k}(s)\right|\right) d s \leq\left|\Delta x_{k}(0)\right| \\
& +\frac{L_{f}}{\Gamma(\alpha)} \int_{0}^{t}(t-s)^{\alpha-1}\left(\left|x_{k+1}(s)-x_{k}(s)\right|\right. \\
& +\left|\sum_{l=1}^{m}\left(x_{k+1}\left(s-\tau_{l}\right)-x_{k}\left(s-\tau_{l}\right)\right)\right| \mid d s \\
& +\frac{L_{I} b_{u}}{\Gamma(\alpha)} \int_{0}^{t}(t-s)^{\alpha-1}\left(\left|x_{k+1}(s)-x_{k}(s)\right|\right.
\end{aligned}
$$

$$
\begin{aligned}
& \left.+\left|\sum_{l=1}^{n}\left(x_{k+1}\left(s-\xi_{l}\right)-x_{k}\left(s-\xi_{l}\right)\right)\right|\right) d s \\
& +\frac{b_{I}}{\Gamma(\alpha)} \int_{0}^{t}(t-s)^{\alpha-1}\left|\Delta u_{k}(s)\right| d s \leq\left|\Delta x_{k}(0)\right| \\
& +\frac{L_{f}}{\Gamma(\alpha)} \int_{0}^{t}(t-s)^{\alpha-1}\left(L_{f}(m+1)+L_{I} b_{u}(n+1)\right) \\
& \cdot\left|\Delta x_{k}(s)\right| d s+\frac{b_{I}}{\Gamma(\alpha)} \int_{0}^{t}(t-s)^{\alpha-1}\left|\Delta u_{k}(s)\right| d s,
\end{aligned}
$$

where $b_{I}, b_{u}$ are defined in $\left(H_{6}\right)$.

Using Lemma 5, we get

$$
\begin{aligned}
& \left|\Delta x_{k}(t)\right| \\
& \leq\left(\left|\Delta x_{k}(0)\right|+\frac{b_{I}}{\Gamma(\alpha)} \int_{0}^{t}(t-s)^{\alpha-1}\left|\Delta u_{k}(s)\right| d s\right) \\
& \cdot e^{c / \Gamma(\alpha) \int_{0}^{t}(t-s)^{\alpha-1} d s} \\
& \leq\left(\left|\Delta x_{k}(0)\right|+\frac{b_{I}}{\Gamma(\alpha)} \int_{0}^{t}(t-s)^{\alpha-1}\left|\Delta u_{k}(s)\right| d s\right) \\
& \cdot e^{c T^{\alpha} / \Gamma(\alpha+1)},
\end{aligned}
$$

where $c=L_{f}(m+1)+L_{I} b_{u}(n+1)$.

We know

$$
\begin{aligned}
e_{k+1}(t)= & e_{k}(t)-\left(y_{k+1}(t)-y_{k}(t)\right) \\
= & e_{k}(t)-g_{x}\left(\mu_{k}(t), t\right) \Delta x_{k}(t) \\
& -d \int_{0}^{t} \Delta u_{k}(s) d s
\end{aligned}
$$

Taking (51) into (64), one obtains

$$
\begin{aligned}
& e_{k+1}(t)=e_{k}(t)-\left(y_{k+1}(t)-y_{k}(t)\right)=e_{k}(t) \\
& -g_{x}\left(\mu_{k}(t), t\right) \Delta x_{k}(t)-d \int_{0}^{t} \Delta u_{k}(s) d s=e_{k}(t) \\
& -g_{x}\left(\mu_{k}(t), t\right) \Delta x_{k}(t)-d\left(\eta_{1} \int_{0}^{t} e_{k}(s) d s\right. \\
& \left.+\eta_{2} \int_{0}^{t} \dot{e}_{k}(s) d s+\eta_{3} \int_{0}^{t} \int_{0}^{s} e_{k}(\tau) d \tau d s\right)=e_{k}(t) \\
& -g_{x}\left(\mu_{k}(t), t\right) \Delta x_{k}(t)-d\left(\eta_{1} \int_{0}^{t} e_{k}(s) d s\right. \\
& \left.+\eta_{2}\left(e_{k}(t)-e_{k}(0)\right)+\eta_{3} \int_{0}^{t} \int_{\tau}^{t} e_{k}(\tau) d s d \tau\right)=(1
\end{aligned}
$$




$$
\begin{array}{ll}
\left.-d \eta_{2}\right) e_{k}(t)-g_{x}\left(\mu_{k}(t), t\right) \Delta x_{k}(t) & +d \eta_{2} e_{k}(0)-g_{x}\left(\mu_{k}(t), t\right) \Delta x_{k}(t) \\
-d\left(\eta_{1} \int_{0}^{t} e_{k}(s) d s-\eta_{2} e_{k}(0)\right. & -d \eta_{1} \int_{0}^{t} e_{k}(s) d s-d \eta_{3} \int_{0}^{t}(t-s) e_{k}(s) d s
\end{array}
$$

$$
\left.+\eta_{3} \int_{0}^{t}(t-s) e_{k}(s) d s\right)=\left(1-d \eta_{2}\right) e_{k}(t)
$$

Combining with (64) and Lemma 8, we can get

$$
\begin{aligned}
& \left|e_{k+1}(t)\right| \leq\left|1-d \eta_{2}\right|\left|e_{k}(t)\right|+\left|d \eta_{2}\right|\left|e_{k}(0)\right|+\xi\left|\Delta x_{k}(t)\right|+\left|d \eta_{1}\right| \int_{0}^{t}\left|e_{k}(s)\right| d s+\left|d \eta_{3}\right| \int_{0}^{t}(t-s)\left\|e_{k}(s)\right\| d s \\
& \leq\left|1-d \eta_{2}\right|\left|e_{k}(t)\right|+\left|d \eta_{2}\right|\left|e_{k}(0)\right|+\xi\left(\left|\Delta x_{k}(0)\right|+\frac{b_{I}}{\Gamma(\alpha)} \int_{0}^{t}(t-s)^{\alpha-1}\left|\Delta u_{k}(s)\right| d s\right) e^{c T^{\alpha} / \Gamma(\alpha+1)}+\left|d \eta_{1}\right| \int_{0}^{t}\left|e_{k}(s)\right| d s+\left|d \eta_{3}\right| \int_{0}^{t}(t-s)\left|e_{k}(s)\right| d s \\
& \leq\left\{\begin{array}{l}
\left|1-d \eta_{2}\right|\left|e_{k}(t)\right|+\left(\left|d \eta_{2}\right|+\xi \varrho e^{c T^{\alpha} / \Gamma(\alpha+1)}\right)\left|e_{k}(0)\right|+\frac{b_{I} \xi e^{c T^{\alpha} / \Gamma(\alpha+1)}}{\Gamma(\alpha)} \cdot \frac{e^{\lambda t^{\alpha}} t^{\alpha}}{\alpha([\lambda]+1)^{\alpha}}\left\|\Delta u_{k}\right\|_{\lambda, \alpha}+\left|d \eta_{1}\right| t e^{\lambda t^{\alpha}}\left\|e_{k}\right\|_{\lambda, \alpha}+\left|d \eta_{3}\right| \cdot \frac{t^{2}}{2} e^{\lambda t^{\alpha}}\left\|e_{k}\right\|_{\lambda, \alpha}, \quad[\lambda]=0, \\
\left|1-d \eta_{2}\right|\left|e_{k}(t)\right|+\left(\left|d \eta_{2}\right|+\xi \varrho e^{c T^{\alpha} / \Gamma(\alpha+1)}\right)\left|e_{k}(0)\right|+\frac{b_{I} \xi e^{c T^{\alpha} / \Gamma(\alpha+1)}}{\Gamma(\alpha)} \cdot \frac{e^{\lambda t^{\alpha}}\left(1+t^{\alpha}\right)}{\alpha[\lambda]^{\alpha}}\left\|\Delta u_{k}\right\|_{\lambda, \alpha}+\left|d \eta_{1}\right| t e^{\lambda t^{\alpha}}\left\|e_{k}\right\|_{\lambda, \alpha}+\left|d \eta_{3}\right| \cdot \frac{t^{2}}{2} e^{\lambda t^{\alpha}}\left\|e_{k}\right\|_{\lambda, \alpha}, \quad[\lambda] \geq 1,
\end{array}\right.
\end{aligned}
$$

where $\xi=\max \left\{\left|\beta_{1}\right|,\left|\beta_{2}\right|\right\}$.

Multiplying $e^{-\lambda t^{\alpha}}$ on both sides of (66) and taking the maximum value on $[0, T]$, we can get

$\left\|e_{k+1}\right\|_{\lambda, \alpha}$

$$
\leq\left\{\begin{array}{l}
\left(\left|1-d \eta_{2}\right|+\left|d \eta_{1}\right| T+\left|d \eta_{3}\right| \cdot \frac{T^{2}}{2}\right)\left\|e_{k}\right\|_{\lambda, \alpha}+\left(\left|d \eta_{2}\right|+\xi \varrho e^{c T^{\alpha} / \Gamma(\alpha+1)}\right)\left\|e_{k}(0)\right\|_{\lambda, \alpha}+\frac{b_{I} \xi e^{c T^{\alpha} / \Gamma(\alpha+1)} \Gamma(\alpha) \cdot T^{\alpha}}{\alpha(1+[\lambda])^{\alpha}}\left\|\Delta u_{k}\right\|_{\lambda, \alpha}, \quad[\lambda]=0, \\
\left(\left|1-d \eta_{2}\right|+\left|d \eta_{1}\right| T+\left|d \eta_{3}\right| \cdot \frac{T^{2}}{2}\right)\left\|e_{k}\right\|_{\lambda, \alpha}+\left(\left|d \eta_{2}\right|+\xi \varrho e^{c T^{\alpha} / \Gamma(\alpha+1)}\right)\left\|e_{k}(0)\right\|_{\lambda, \alpha}+\frac{b_{I} \xi e^{c T^{\alpha} / \Gamma(\alpha+1)} \Gamma(\alpha) \cdot\left(1+T^{\alpha}\right)}{\alpha[\lambda]^{\alpha}}\left\|\Delta u_{k}\right\|_{\lambda, \alpha}, \quad[\lambda] \geq 1 .
\end{array}\right.
$$

Set

$$
\begin{gathered}
\mu=\max \left\{\frac{b_{I} \xi e^{c T^{\alpha} / \Gamma(\alpha+1)} \Gamma(\alpha) \cdot T^{\alpha}}{\alpha(1+[\lambda])^{\alpha}},\right. \\
\left.\frac{b_{I} \xi e^{c T^{\alpha} / \Gamma(\alpha+1)} \Gamma(\alpha) \cdot\left(1+T^{\alpha}\right)}{\alpha[\lambda]^{\alpha}}\right\} .
\end{gathered}
$$

There exists a sufficiently large $\lambda$ such that $\mu$ is very small and using (56) and (60) we can derive $\lim _{k \rightarrow \infty}\left\|e_{k}\right\|_{\lambda, \alpha}=0$. The proof is completed.

4.2. Closed-Loop Case. For system (2), consider the closeloop PID-type ILC updating law with initial state learning:

$$
\begin{aligned}
x_{k+1}(0)= & x_{k}(0)+\zeta e_{k+1}(0), \\
u_{k+1}(t)= & u_{k}(t)+\kappa_{1} e_{k+1}(t)+\kappa_{2} \dot{e}_{k+1}(t) \\
& +\kappa_{3} \int_{0}^{t} e_{k+1}(s) d s,
\end{aligned}
$$

where $\varrho$ and $\kappa_{i}(i=1,2,3)$ are unknown parameters to be determined.
Theorem 18. Assume that $\left(H_{1}\right)-\left(H_{5}\right)$ hold. If

$$
\begin{aligned}
& \min \left\{\left|1+\beta_{1} \zeta\right|,\left|1+\beta_{2} \zeta\right|\right\} \geq \bar{r}_{0}>1, \\
& r_{2}:=\left|1+d \kappa_{2}\right|-\left|d \kappa_{1}\right|-\frac{\left|d \kappa_{3}\right| T^{2}}{2}>1,
\end{aligned}
$$

then the nonlinear multiple time-delays system (2) with the close-loop PID-type ILC updating law (69) guarantees that $y_{k}$ tends to $y_{d}$ as $k \rightarrow \infty$ in the sense of $\lambda, \alpha$-norm for all $t \in$ $[0, T]$, where $\psi_{d}(t)$ is the desired initial state on $[-a, 0]$ and $y_{d}(t)$ is the desired output trajectory.

Proof. Note that

$$
\begin{aligned}
e_{k+1}(t)= & e_{k}(t)+g_{k}(t)-g_{k+1}(t) \\
= & e_{k}(t)-g_{x}\left(\mu_{k}(t), t\right) \Delta x_{k}(t) \\
& -d \int_{0}^{t} \Delta u_{k}(s) d s,
\end{aligned}
$$

where $\mu_{k}(t)$ lies in the segment with end points $x_{k}(t)$ and $x_{k+1}(t)$ for $t \in[0, T]$.

In what follows, we show that $\left\|e_{k}\right\|_{\lambda, \alpha} \rightarrow 0$ as $k \rightarrow \infty$ for a.e. $t \in[0, T]$. By using mean value theorem we have

$$
\begin{aligned}
e_{k+1}(0) & =e_{k}(0)+y_{k}(0)-y_{k+1}(0) \\
& =e_{k}(0)-g_{x}\left(\mu_{k}(0), 0\right) \Delta x_{k}(0) .
\end{aligned}
$$


Substituting (69) into (73) and taking the absolute value, we have

$$
\left|1+g_{x}\left(\mu_{k}(0), 0\right) \zeta\right|\left|e_{k+1}(0)\right|=\left|e_{k}(0)\right|
$$

It follows from (70) that

$$
\lim _{k \rightarrow \infty}\left\|e_{k}(0)\right\|_{\lambda, \alpha}=0
$$

Similar to the proof of Theorem 17, we can get

$$
\begin{aligned}
& e_{k+1}(t)=e_{k}(t)-\left(y_{k+1}(t)-y_{k}(t)\right)=e_{k}(t) \\
& \quad-g_{x}\left(\mu_{k}(t), t\right) \Delta x_{k}(t)-d \int_{0}^{t} \Delta u_{k}(s) d s=e_{k}(t)
\end{aligned}
$$

$$
\begin{aligned}
& -g_{x}\left(\mu_{k}(t), t\right) \Delta x_{k}(t)-d\left(\kappa_{1} \int_{0}^{t} e_{k+1}(s) d s\right. \\
& \left.+\kappa_{2} \int_{0}^{t} \dot{e}_{k+1}(s) d s+\kappa_{3} \int_{0}^{t} \int_{0}^{s} e_{k+1}(\tau) d \tau d s\right) \\
& =e_{k}(t)-g_{x}\left(\mu_{k}(t), t\right) \Delta x_{k}(t) \\
& -d\left(\kappa_{1} \int_{0}^{t} e_{k+1}(s) d s+\kappa_{2}\left(e_{k+1}(t)-e_{k+1}(0)\right)\right. \\
& \left.+\kappa_{3} \int_{0}^{t} \int_{\tau}^{t} e_{k}(\tau) d s d \tau\right)=e_{k}(t)-d \kappa_{2} e_{k+1}(t) \\
& +d \kappa_{2} e_{k+1}(0)-g_{x}\left(\mu_{k}(t), t\right) \Delta x_{k}(t) \\
& -d \kappa_{1} \int_{0}^{t} e_{k+1}(s) d s-d \kappa_{3} \int_{0}^{t}(t-s) e_{k+1}(s) d s .
\end{aligned}
$$

Then, one has

$$
\begin{aligned}
& \left|1+d \kappa_{2}\right|\left|e_{k+1}(t)\right| \leq\left|e_{k}(t)\right|+\left|d \kappa_{2}\right|\left|e_{k+1}(0)\right|+\xi\left|\Delta x_{k}(t)\right|+\left|d \kappa_{1}\right| \int_{0}^{t}\left|e_{k+1}(s)\right| d s+\left|d \kappa_{3}\right| \int_{0}^{t}(t-s)\left|e_{k+1}(s)\right| d s \\
& \leq\left|e_{k}(t)\right|+\left|d \kappa_{2}\right|\left|e_{k+1}(0)\right|+\xi\left(\left|\Delta x_{k}(0)\right|+\frac{b_{I}}{\Gamma(\alpha)} \int_{0}^{t}(t-s)^{\alpha-1}\left|\Delta u_{k}(s)\right| d s\right) e^{c T^{\alpha} / \Gamma(\alpha+1)}+\left|d \kappa_{1}\right| \int_{0}^{t}\left|e_{k+1}(s)\right| d s+\left|d \kappa_{3}\right| \int_{0}^{t}(t-s)\left|e_{k+1}(s)\right| d s \\
& \leq\left|e_{k}(t)\right|+\left(\left|d \kappa_{2}\right|+\xi \zeta e^{c T^{\alpha} / \Gamma(\alpha+1)}\right)\left|e_{k+1}(0)\right|+\xi \cdot e^{c T^{\alpha} / \Gamma(\alpha+1)} \frac{b_{I}}{\Gamma(\alpha)} \int_{0}^{t}(t-s)^{\alpha-1} e^{\lambda s^{\alpha}} d s\left\|\Delta u_{k}\right\|_{\lambda, \alpha}+\left|d \kappa_{1}\right| \int_{0}^{t} e^{\lambda s^{\alpha}} d s\left\|e_{k+1}\right\|_{\lambda, \alpha} \\
& \\
& +\left|d \kappa_{3}\right| \int_{0}^{t}(t-s) e^{\lambda s^{\alpha}} d s\left\|e_{k+1}\right\|_{\lambda, \alpha} \\
& \leq\left\{\begin{array}{l}
\left|e_{k}(t)\right|+\left(\left|d \kappa_{2}\right|+\xi \zeta e^{c T^{\alpha} / \Gamma(\alpha+1)}\right)\left|e_{k+1}(0)\right|+\frac{b_{I} \xi e^{c T^{\alpha} / \Gamma(\alpha+1)}}{\Gamma(\alpha)} \cdot \frac{e^{\lambda t^{\alpha}} t^{\alpha}}{\alpha(1+[\lambda])^{\alpha}}\left\|\Delta u_{k}\right\|_{\lambda, \alpha}+\left|d \kappa_{1}\right| t e^{\lambda t^{\alpha}}\left\|e_{k+1}\right\|_{\lambda, \alpha}+\left|d \kappa_{3}\right| \cdot \frac{t^{2}}{2} e^{\lambda t^{\alpha}}\left\|e_{k+1}\right\|_{\lambda, \alpha}, \quad[\lambda]=0, \\
\left|e_{k}(t)\right|+\left(\left|d \kappa_{2}\right|+\xi \zeta e^{c T^{\alpha} / \Gamma(\alpha+1)}\right)\left|e_{k+1}(0)\right|+\frac{b_{I} \xi e^{c T^{\alpha} / \Gamma(\alpha+1)}}{\Gamma(\alpha)} \cdot \frac{e^{\lambda t^{\alpha}}\left(1+t^{\alpha}\right)}{\alpha[\lambda]^{\alpha}}\left\|\Delta u_{k}\right\|_{\lambda, \alpha}+\left|d \kappa_{1}\right| t e^{\lambda t^{\alpha}}\left\|e_{k+1}\right\|_{\lambda, \alpha}+\left|d \kappa_{3}\right| \cdot \frac{t^{2}}{2} e^{\lambda t^{\alpha}}\left\|e_{k+1}\right\|_{\lambda, \alpha}, \quad[\lambda] \geq 1,
\end{array}\right.
\end{aligned}
$$

where $\xi=\max \left\{\left|\beta_{1}\right|,\left|\beta_{2}\right|\right\}$.

Multiplying $e^{-\lambda t^{\alpha}}$ on both sides of (77) and taking $\lambda, \alpha$ norm, we can derive

$$
\begin{aligned}
& \left|1+d \kappa_{2}\right|\left\|e_{k+1}\right\|_{\lambda, \alpha} \\
& \leq\left\{\begin{array}{l}
\left\|e_{k}\right\|_{\lambda, \alpha}+\left(\left|d \kappa_{2}\right|+\xi \zeta e^{c T^{\alpha} / \Gamma(\alpha+1)}\right)\left\|e_{k+1}(0)\right\|_{\lambda, \alpha}+\frac{b_{I} \xi e^{c T^{\alpha} / \Gamma(\alpha+1)}}{\Gamma(\alpha)} \cdot \frac{T^{\alpha}}{\alpha(1+[\lambda])^{\alpha}}\left\|\Delta u_{k}\right\|_{\lambda, \alpha}+\left|d \kappa_{1}\right| T\left\|e_{k+1}\right\|_{\lambda, \alpha}+\left|d \kappa_{3}\right| \cdot \frac{T^{2}}{2}\left\|e_{k+1}\right\|_{\lambda, \alpha}, \quad[\lambda]=0, \\
\left\|e_{k}\right\|_{\lambda, \alpha}+\left(\left|d \kappa_{2}\right|+\xi \zeta e^{c T^{\alpha} / \Gamma(\alpha+1)}\right)\left\|e_{k+1}(0)\right\|_{\lambda, \alpha}+\frac{b_{I} \xi e^{c T^{\alpha} / \Gamma(\alpha+1)}}{\Gamma(\alpha)} \cdot \frac{\left(1+T^{\alpha}\right)}{\alpha[\lambda]^{\alpha}}\left\|\Delta u_{k}\right\|_{\lambda, \alpha}+\left|d \kappa_{1}\right| T\left\|e_{k+1}\right\|_{\lambda, \alpha}+\left|d \kappa_{3}\right| \cdot \frac{T^{2}}{2}\left\|e_{k+1}\right\|_{\lambda, \alpha}, \quad[\lambda] \geq 1 .
\end{array}\right. \\
& \widehat{\mu}=\max \left\{\frac{b_{I} \xi e^{c T^{\alpha} / \Gamma(\alpha+1)}}{\Gamma(\alpha)} \cdot \frac{T^{\alpha}}{\alpha(1+[\lambda])^{\alpha}}, \frac{b_{I} \xi e^{c T^{\alpha} / \Gamma(\alpha+1)}}{\Gamma(\alpha)}\right. \\
& \left.\frac{\left(1+T^{\alpha}\right)}{\alpha[\lambda]^{\alpha}}\right\}
\end{aligned}
$$

There exists a sufficiently large $\lambda$ such that $\widehat{\mu}$ is very small and using (71) and (75) we can derive $\lim _{k \rightarrow \infty}\left\|e_{k}\right\|_{\lambda, \alpha}=0$. This completes the proof of Theorem 18.

\section{Examples}

In this section, we give two examples to illustrate our results above. 
Example 1. Let $\alpha=1 / 2, L_{f}=1 / 8, L_{I}=1 / 12, m=2, n=1$, and $T=0.2$. We consider the following nonlinear fractional multiple time-delays differential equations

$$
\begin{aligned}
{ }^{c} D_{0+}^{1 / 2} x(t)= & \frac{1}{8} \sin (5 x(t))+\frac{1}{8} \frac{x^{2}(t-1)}{1+x^{2}(t-1)} \\
& +\frac{1}{8} \cos \left(x\left(t-\frac{1}{2}\right)\right) \\
& +\frac{1}{12} \sin \left(3 x\left(t-\frac{1}{3}\right)\right) u(t), \\
x(0)= & 1, \quad t \in[0,0.2],
\end{aligned}
$$

and the inequalities

$$
\begin{gathered}
\mid{ }^{c} D_{t}^{1 / 2} z(t)-f\left(t, z(t) z(t-1), z\left(t-\frac{1}{2}\right)\right) \\
+I\left(t, z\left(t-\frac{1}{3}\right)\right) u(t) \mid \leq \epsilon, \\
\mid{ }^{c} D_{t}^{1 / 2} z(t)-f\left(t, z(t) z(t-1), z\left(t-\frac{1}{2}\right)\right) \\
+I\left(t, z\left(t-\frac{1}{3}\right)\right) u(t) \mid \leq \epsilon E_{1 / 2}\left(t^{1 / 2}\right) .
\end{gathered}
$$

Define $f(t, x(t), x(t-1), x(t-1 / 2))=(1 / 8) \sin (5 x(t))+$ $(1 / 8)\left(x^{2}(t-1) /\left(1+x^{2}(t-1)\right)\right)+(1 / 8) \cos (x(t-1 / 2))$ and $I(t, x(t-1 / 3))=(1 / 12) \sin (3 x(t-1 / 3))$ and set $b_{u}=1, \lambda=$ 4.2. Thus $c=0.5417$; then $c\left(1+T^{\alpha}\right) / \Gamma(\alpha+1)[\lambda]^{\alpha}=0.4423<$ 1. Now all the assumptions in Theorems 9,12 , and 15 are satisfied, problem (80) has a unique solution, and the first equation in (80) is Ulam-Hyers stable with $|z(t)-x(t)| \leq$ $c \epsilon, t \in[-1,0.2]$ and Ulam-Hyers-Mittag-Leffler stable with

$$
|z(t)-x(t)| \leq c_{E_{1 / 2}} \in E_{1 / 2}\left(t^{1 / 2}\right), \quad t \in[-1,0.2],
$$

where $c=26.9701, c_{E_{1 / 2}}=1.3447$.

Example 2. Let $\alpha=1 / 2, L_{f}=1 / 8, L_{I}=1 / 12, m=2, n=1$, and $T=0.2$. We consider the following nonlinear fractional multiple time-delays differential equations

$$
\begin{aligned}
{ }^{c} D_{0+}^{1 / 2} x_{k}(t)= & \frac{1}{8} \sin \left(5 x_{k}(t)\right)+\frac{1}{8} \frac{x_{k}^{2}(t-1)}{1+x_{k}^{2}(t-1)} \\
& +\frac{1}{8} \cos \left(x_{k}\left(t-\frac{1}{2}\right)\right) \\
& +\frac{1}{12} \sin \left(3 x_{k}\left(t-\frac{1}{3}\right)\right) u_{k}(t), \\
x(0)= & 1, \quad t \in[-1,0], \quad t 0.2], \\
y_{k}(t)= & 2 x_{k}(t)+\frac{3}{2} \sin x_{k}(t)+2 \int_{0}^{t} u_{k}(s) d s,
\end{aligned}
$$

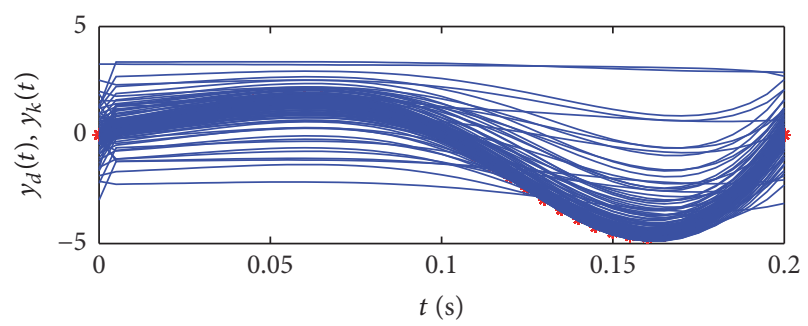

(a)

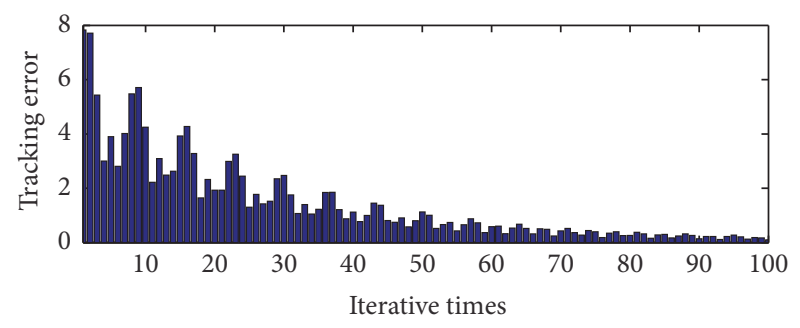

(b)

FIGURE 1: The system output $y_{k}(t)$ (blue line), the desired trajectory $y_{d}(t)$ (red line), and the tracking error for (83).

and the desired reference trajectory $y_{d}(t)=10 \sin (10 \pi t)(1+$ $\cos (10 \pi t))+2, t \in[0,0.2]$.

Consider problem (83) and the open-loop PID-type ILC updating law with initial state learning:

$$
\begin{aligned}
& x_{k+1}(0)=x_{k}(0)+\frac{1}{5} e_{k}(0), \\
& u_{k+1}(t)=u_{k}(t)+\frac{1}{10} e_{k}(t)+\frac{1}{2} \dot{e}_{k}(t)+\frac{1}{5} \int_{0}^{t} e_{k}(s) d s .
\end{aligned}
$$

Obviously, $r_{0}=0.9<1$ and $r_{1}=0.048<1$. All the conditions of Theorem 17 are satisfied.

Next, consider problem (83) and the close-loop PID-type ILC updating law with initial state learning:

$$
\begin{aligned}
x_{k+1}(0)= & x_{k}(0)+\frac{3}{2} e_{k+1}(0), \\
u_{k+1}(t)= & u_{k}(t)+\frac{3}{2} e_{k+1}(t)+\frac{5}{2} \dot{e}_{k+1}(t) \\
& +6 \int_{0}^{t} e_{k+1}(s) d s .
\end{aligned}
$$

Thus $\bar{r}_{0}=1.75>1$ and $r_{2}=2.76>1$. All the conditions of Theorem 18 are satisfied.

Numerical simulation diagram for the open-loop control is shown in Figure 1(a).

Figure 1(b) shows the supremum norm of the tracking error in each iteration and the 100th error is 0.0979 .

\section{Conclusions}

This paper is twofold: in the first part we show the existence and uniqueness result and present Ulam-Hyers and UlamHyers-Mittag-Leffler stability results for fractional nonlinear 
multiple time-delays systems. In the second part we apply PID-type learning updating laws to obtain sequences of tracking trajectory to approximate a given trajectory. In the future, we will study the related topic of noninstantaneous impulsive systems [48].

\section{Conflicts of Interest}

The authors declare that they have no conflicts of interest.

\section{Acknowledgments}

The first and second author's work was partially supported by National Natural Science Foundation of China (11661016), Training Object of High Level and Innovative Talents of Guizhou Province ((2016)4006), Unite Foundation of Guizhou Province ([2015]7640), and Graduate ZDKC([2015]003). The third author acknowledges the support by National Natural Science Foundation of China (11671339). The authors are grateful to Associate Professor Dr. Qian Chen for her discussion, careful reading of the manuscript, and valuable comments.

\section{References}

[1] K. S. Miller and B. Ross, An Introduction to the Fractional Calculus and Differential Equations, John Wiley, Hoboken, NJ, USA, 1993.

[2] A. A. Kilbas, H. M. Srivastava, and J. J. Trujillo, Theory and Applications of Fractional Differential Equations, New York, NY, USA, Elsevier, 2006.

[3] S. Abbas, M. Benchohra, and G. M. N'Guérékata, Topics in Fractional Differential Equations, Springer, New York, NY, USA, 2012.

[4] J. Wang, M. Fečkan, and Y. Zhou, "A survey on impulsive fractional differential equations," Fractional Calculus and Applied Analysis, vol. 19, no. 4, pp. 806-831, 2016.

[5] J. Wang and Y. Zhou, "Analysis of nonlinear fractional control systems in Banach spaces," Nonlinear Analysis. Theory, Methods \& Applications, vol. 74, no. 17, pp. 5929-5942, 2011.

[6] R. Agarwal, D. O’Regan, and S. Hristova, "Stability of Caputo fractional differential equations by Lyapunov functions," Applications of Mathematics, vol. 60, no. 6, pp. 653-676, 2015.

[7] Q. Wang, D. Lu, and Y. Fang, "Stability analysis of impulsive fractional differential systems with delay," Applied Mathematics Letters. An International Journal of Rapid Publication, vol. 40, pp. 1-6, 2015.

[8] I. Stamova and G. Stamov, "Stability analysis of impulsive functional systems of fractional order," Communications in Nonlinear Science and Numerical Simulation, vol. 19, no. 3, pp. 702-709, 2014.

[9] J. Wang and Y. Zhang, "On the concept and existence of solutions for fractional impulsive systems with Hadamard derivatives," Applied Mathematics Letters. An International Journal of Rapid Publication, vol. 39, pp. 85-90, 2015.

[10] J. Wang, A. G. Ibrahim, and M. Fečkan, "Nonlocal impulsive fractional differential inclusions with fractional sectorial operators on Banach spaces," Applied Mathematics and Computation, vol. 257, pp. 103-118, 2015.
[11] M. Li and J. Wang, "Finite time stability of fractional delay differential equations," Applied Mathematics Letters. An International Journal of Rapid Publication, vol. 64, pp. 170-176, 2017.

[12] J. Wang, M. Fečkan, and Y. Zhou, "Center stable manifold for planar fractional damped equations," Applied Mathematics and Computation, vol. 296, pp. 257-269, 2017.

[13] J. Wang, M. Fečkan, and Y. Zhou, "Fractional Order Differential Switched Systems with Coupled Nonlocal Initial and Impulsive Conditions," Bulletin des Sciences Mathématiques, 2017.

[14] S. Abbas, M. Benchohra, M. Rivero, and J. . Trujillo, "Existence and stability results for nonlinear fractional order Riemann-Liouville Volterra-Stieltjes quadratic integral equations," Applied Mathematics and Computation, vol. 247, pp. 319328, 2014.

[15] J. Wang, M. Fečkan, and Y. Zhou, "Ulam's type stability of impulsive ordinary differential equations," Journal of Mathematical Analysis and Applications, vol. 395, no. 1, pp. 258-264, 2012.

[16] S. Tang, A. Zada, S. Faisal, M. M. El-Sheikh, and T. Li, "Stability of higher-order nonlinear impulsive differential equations," Journal of Nonlinear Science and its Applications. JNSA, vol. 9, no. 6, pp. 4713-4721, 2016.

[17] J. Wang, Y. Zhou, and M. Fečkan, "Nonlinear impulsive problems for fractional differential equations and Ulam stability," Computers \& Mathematics with Applications. An International Journal, vol. 64, no. 10, pp. 3389-3405, 2012.

[18] A. Zada, O. Shah, and R. Shah, "Hyers-Ulam stability of nonautonomous systems in terms of bounded-ness of Cauchy problems," Applied Mathematics and Computation, vol. 271, pp. 512-518, 2015.

[19] Y. Zhou, "Attractivity for fractional differential equations in Banach space," Applied Mathematics Letters. An International Journal of Rapid Publication, vol. 75, pp. 1-6, 2018.

[20] Y.Zhou, B. Ahmad, and A. Alsaedi, "Existence of nonoscillatory solutions for fractional neutral differential equations," Applied Mathematics Letters, vol. 72, pp. 70-74, 2017.

[21] Y. Zhou and L. Peng, "Weak solutions of the time-fractional Navier-Stokes equations and optimal control," Computers \& Mathematics with Applications. An International Journal, vol. 73, no. 6, pp. 1016-1027, 2017.

[22] Y. Zhou and L. Peng, "On the time-fractional Navier-Stokes equations," Computers \& Mathematics with Applications. An International Journal, vol. 73, no. 6, pp. 874-891, 2017.

[23] Y. Zhou, V. Vijayakumar, and R. Murugesu, "Controllability for fractional evolution inclusions without compactness," Evolution Equations and Control Theory, vol. 4, no. 4, pp. 507-524, 2015.

[24] Y. Zhou and L. Zhang, "Existence and multiplicity results of homoclinic solutions for fractional Hamiltonian systems," Computers \& Mathematics with Applications. An International Journal, vol. 73, no. 6, pp. 1325-1345, 2017.

[25] Y. Zhou, L. Peng, B. Ahmad, and A. Alsaedi, "Topological properties of solution sets of fractional stochastic evolution inclusions," Advances in Difference Equations, p. 90, 2017.

[26] X. Yang, C. Li, T. Huang, and Q. Song, "Mittag-Leffler stability analysis of nonlinear fractional-order systems with impulses," Applied Mathematics and Computation, vol. 293, pp. 416-422, 2017.

[27] X. Yang, C. Li, Q. Song, T. Huang, and X. Chen, "Mittag-Leffler stability analysis on variable-time impulsive fractional-order neural networks," Neurocomputing, vol. 207, pp. 276-286, 2015.

[28] A. Wu, L. Liu, T. Huang, and Z. Zeng, "Mittag-Leffler stability of fractional-order neural networks in the presence of generalized 
piecewise constant arguments," Neural Networks, vol. 85, pp. 118-127, 2017.

[29] T. Huang, C. Li, S. Duan, and J. A. Starzyk, "Robust exponential stability of uncertain delayed neural networks with stochastic perturbation and impulse effects," IEEE Transactions on Neural Networks and Learning Systems, vol. 23, no. 6, pp. 866-875, 2012.

[30] M. Uchiyama, "Formulation of high-speed motion pattern of a mechanical arm by trial," Transactions of the Society of Instrument and Control Engineers, vol. 14, pp. 706-712, 1978.

[31] S. Arimoto, S. Kawamura, and F. Miyazaki, "Bettering operation of robots by learning," Journal of Robotic Systems, vol. 1, no. 2, pp. 123-140, 1984.

[32] Y. Chen and C. Wen, Iterative learning control, vol. 248 of Lecture Notes in Control and Information Sciences, SpringerVerlag, London, UK, 1999.

[33] M. Norrlof, "Iterative learning control: Analysis, Design, and Experiments, Linköping Studies," in Science and Technology, Dissertations, vol. 653, 2000.

[34] H. S. Ahn, Y. Q. Chen, and K. L. Moore, Iterative Learning Control, Springer, Berlin, Germany, 2007.

[35] J. X. Xu, "A survey on iterative learning control for nonlinear systems," International Journal of Control, vol. 84, no. 7, pp. 1275-1294, 2011.

[36] X. Ruan, Z. Z. Bien, and Q. Wang, "Convergence characteristics of proportional-type iterative learning control in the sense of Lebesgue-p norm," IET Control Theory and Applications, vol. 6, no. 5, pp. 707-714, 2012.

[37] S. Liu, A. Debbouche, and J. Wang, "On the iterative learning control for stochastic impulsive differential equations with randomly varying trial lengths," Journal of Computational and Applied Mathematics, vol. 312, pp. 47-57, 2017.

[38] X. Yu, A. Debbouche, and J. Wang, "On the iterative learning control of fractional impulsive evolution equations in Banach spaces," Mathematical Methods in the Applied Sciences, 2015.

[39] D. H. Owens, "Norm Optimal Iterative Learning Control," in Iterative Learning Control, Advances in Industrial Control, pp. 233-276, Springer, London, UK, 2016.

[40] L. M. Hideg, "Time delays in iterative learning control schemes," in Proceedings of the 10th IEEE International Symposium on Intelligent Control, pp. 215-220, August 1995.

[41] B. Zhang, G. Tang, and S. Zheng, "PD-type iterative learning control for nonlinear time-delay system with external disturbance," Journal of Systems Engineering and Electronics, vol. 17, no. 3, pp. 600-605, 2006.

[42] F. Ma, C. Li, and T. Huang, "Iterative learning control design of nonlinear multiple time-delay systems," Applied Mathematics and Computation, vol. 218, no. 8, pp. 4333-4340, 2011.

[43] I. A. Rus, "Gronwall lemmas: ten open problems," Scientiae Mathematicae Japonicae, vol. 70, pp. 221-228, 2009.

[44] Y. Li, H.-S. Ahn, and Y. Chen, "Iterative learning control of a class of fractional order nonlinear systems," in Proceedings of the 2010 IEEE International Symposium on Intelligent Control, ISIC 2010, pp. 779-782, September 2010.

[45] Y. Zhang and J. R. Wang, "Nonlocal Cauchy problems for a class of implicit impulsive fractional relaxation differential systems," Journal of Applied Mathematics and Computing, vol. 52, no. 1-2, pp. 323-343, 2016.

[46] J. Wang and X. Li, " $E_{\alpha}$-Ulam type stability of fractional order ordinary differential equations," Applied Mathematics and Computation, vol. 45, no. 1-2, pp. 449-459, 2014.
[47] J. Wang and Y. Zhang, "Ulam-Hyers-Mittag-Leffler stability of fractional-order delay differential equations," Optimization. A Journal of Mathematical Programming and Operations Research, vol. 63, no. 8, pp. 1181-1190, 2014.

[48] J. Wang, M. Fečkan, and Y. Tian, "Stability analysis for a general class of non-instantaneous impulsive differential equations," Mediterranean Journal of Mathematics, vol. 14, no. 2, Art. 46, 21 pages, 2017. 


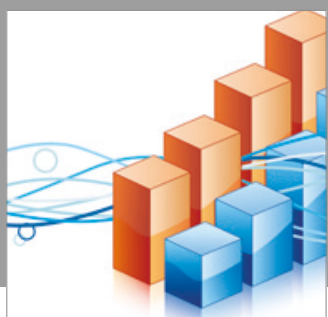

Advances in

Operations Research

vatersals

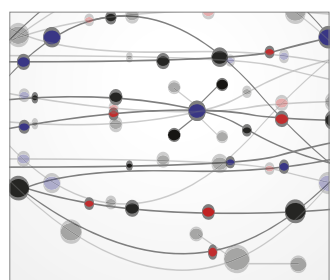

\section{The Scientific} World Journal
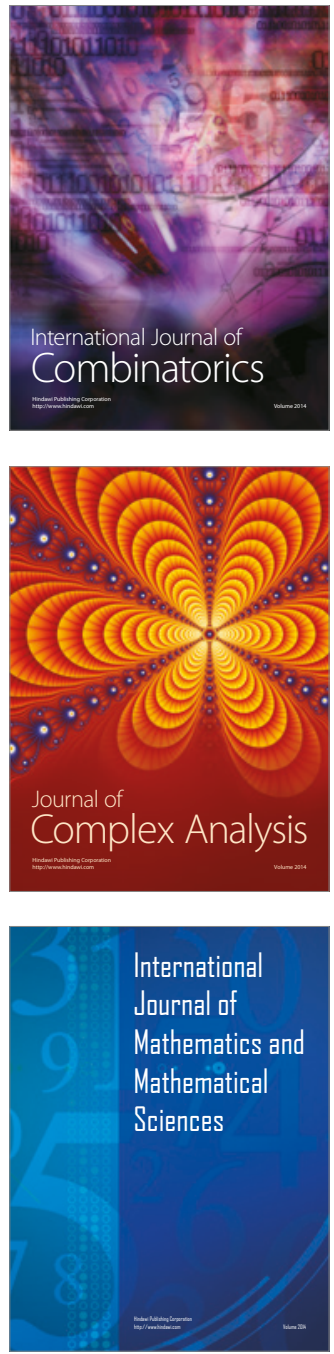
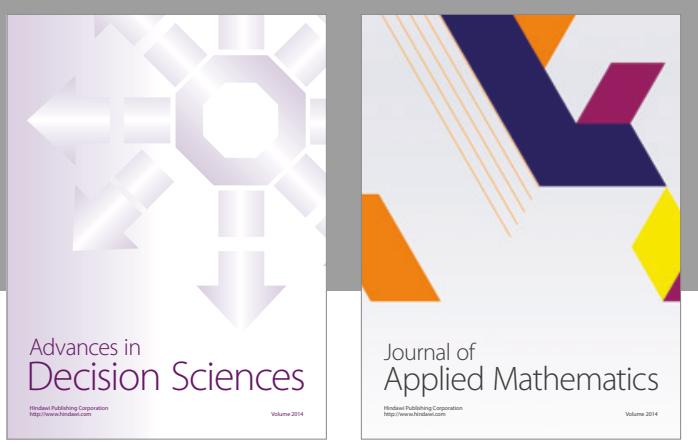

Algebra

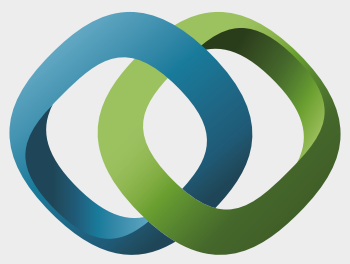

\section{Hindawi}

Submit your manuscripts at

https://www.hindawi.com
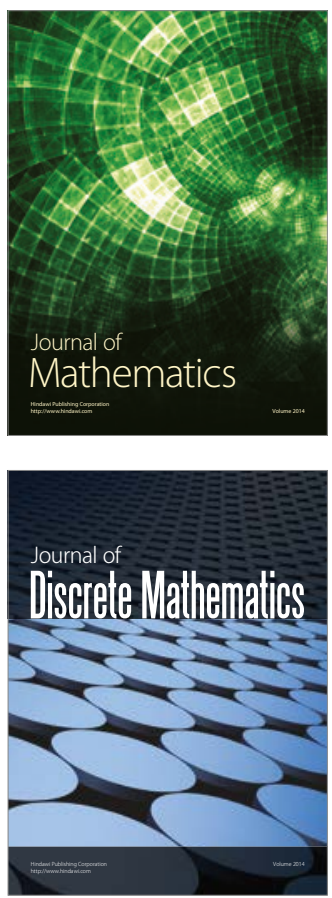

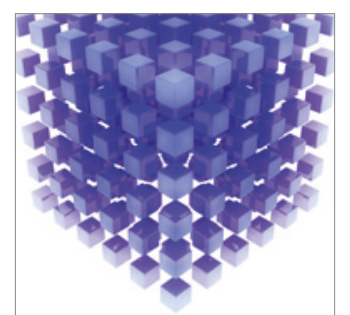

Mathematical Problems in Engineering
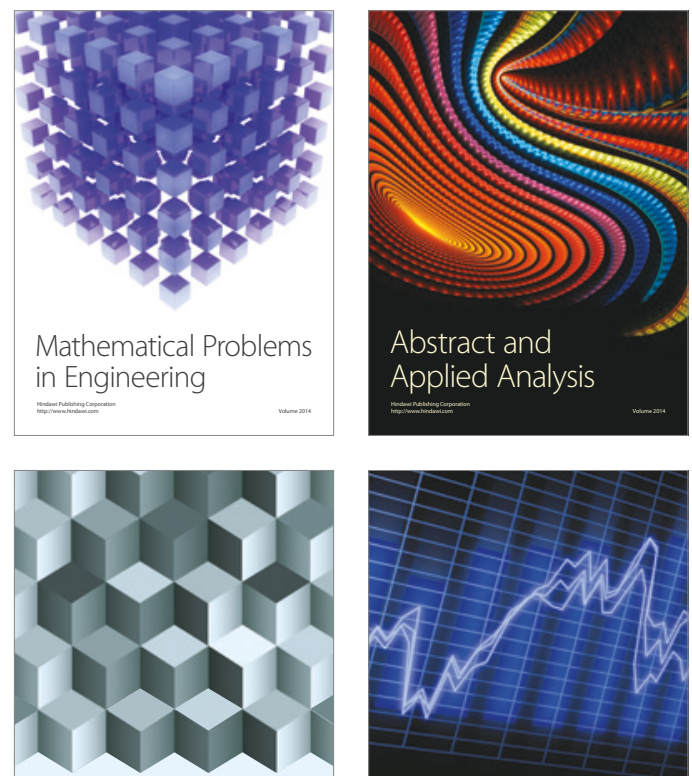

Journal of

Function Spaces

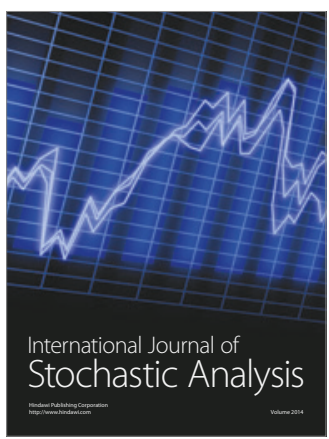

Probability and Statistics
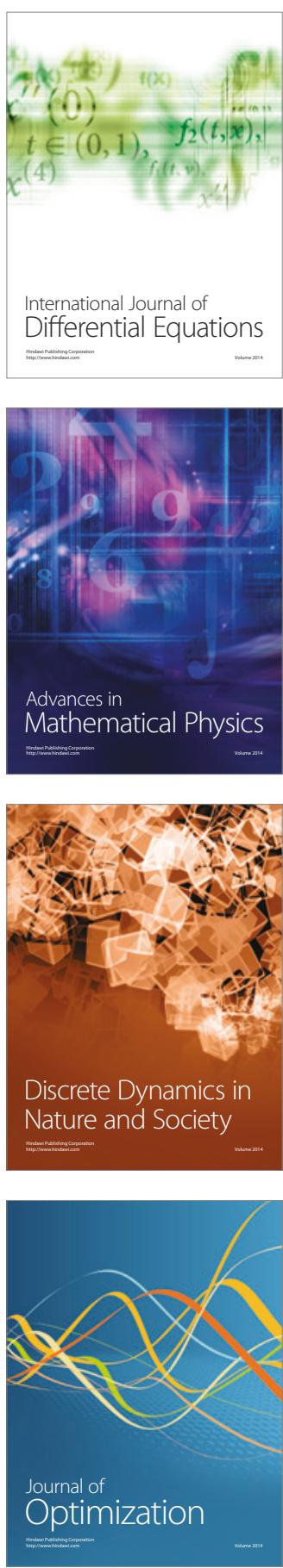